Ann. Biol. anim. Bioch. Biophys., 1979, 19 (3 B), 849-879.

\title{
Activités de la musculature gastro-intestinale et mouvements des contenus digestifs
}

par J. P. LAPLACE, C. ROMAN*

\author{
Laboratoire de Physiologie de la Nutrition \\ I.N.R.A., 78350 Jouy-en-Josos \\ * Laboratoire de Neurophysiologie Générale \\ Faculté des Sciences ef techniques Saint-Jérôme \\ 13397 Marseille Cedex 4.
}

Summary. Gastro-infestinal muscle activities and movements of digesta.

A brief survey is made of the ionic bases of the activity of the smooth muscle cell : variations of membrane potential, mechanical activation and conduction of excitation through the nexuses (gap junctions). The different components of longitudinal smooth muscle electrical activity are reviewed for the gastric antrum and the small intestine, i.e. fibers with oscillating membrane potentials. Gastric (plateau potentials, i. e. electrical control activity possibly followed by response activity) and intestinal (slow waves possibly with superimposed spikes) activities are described. The origin and propagation of periodical oscillations (gastric control activities, intestinal slow waves) are briefly recalled. The role of these oscillations in the control of gastro-intestinal motility is then developed. The coordination of circular and longitudinal muscle layer activities is defined as well as the relative importance of the contraction of each layer with respect to endoluminal pressures. The organization of these activities as related to different types of gastric contractions and intestinal movements is explained. The motility pattern, in the form of myoelectric migrating complexes, is described for the whole tract. The relationship between antral contractions and gastric emptying and the possible role of pendular intestinal movements are evoked, as well as the contribution of intestinal contractions resulting from the coordinated activity of the two muscle layers. The significance of local contractions, propagated contractions, and their regrouping into different phases of the myoelectric complexes is discussed on the basis of recent data. The relative importance of myogenic events, inducing the rhythmicity and polarity of gastro-intestinal movements, is emphasized in the conclusion. However, the role of the intraparietal nervous system in coordinating the activity of the two muscle layers during peristaltism is also pointed out. The myoelectric migrating complexes, regulating food passage in the digestive tract, probably require a co-participation of the intrinsic and extrinsic nervous systems. The neurogenous and humoral influences contribute to adjusting motor events to various feeding conditions. Lastly, as a part of the fast electrical activities of the intestine (spikes) is not accompanied by measurable food passage, the propulsion of intestinal contents does not seem to be the sole function of intestinal motility.

Les aspects moteurs du fonctionnement du tube digestif sont bien souvent étudiés par des groupes scientifiques qui diffèrent largement quant à leurs préoccupations - physiologie du muscle lisse vs. mouvements des contenus digestifs - quant à leurs 
motivations - connaissance fondamentale ou application clinique vs. nutrition animale - et évidemment quant aux techniques mises en œuvre. Pourtant, motricité et transit digestifs sont un seul et même aspect du fonctionnement gastro-intestinal. Pendant de nombreuses années, la relation de cause à effet entre motricité de la paroi et déplacement des contenus endoluminaux, globalement évidente, a été presque exclusivement implicite. Nous nous proposons ici, non pas de procéder à une revue exhaustive des connaissances dans ce domaine, mais d'exposer de façon aussi simple que possible et sur la base d'un certain nombre de données récentes, quelle est la nature de cette relation entre motricité et transit dans le cas de l'estomac et de l'intestin grêle. Le lecteur trouvera annexé à ce fexte un glossaire des termes essentiels, de leurs abréviations, de leurs équivalents anglo-saxons ef éventuellement de certains de leurs synonymes.

\section{1. - Structure et contraction de la cellule musculaire lisse.}

La motricité de l'estomac et de l'intestin grêle est assurée chez les Mammifères par la contraction coordonnée de deux couches musculaires, l'une longitudinale externe, l'autre circulaire interne, ef toutes deux constituées de fibres lisses.

Au plan morphologique, la cellule musculaire lisse, fusiforme $(200 \mu \mathrm{m}$ de longueur pour 2 à $7 \mu \mathrm{m}$ de diamètre au plus) a fait l'objet de descriptions détaillées (voir Dewey ef Barr, 1968 ; Huddart et Hunt, 1975 ; Marechal et Casteels, 1976). L'agencement des protéines contractiles filamenteuses à l'intérieur de la cellule, et les modifications de cette structure lors de la contraction (glissement des filaments les uns sur les autres conduisant au raccourcissement), constituent un sujet riche de controverses (Needham et Shoenberg, 1968 ; Bülbring et Needham, 1973 ; Siegman, 1975). Cet agencement des différents types de filaments pourrait être le responsable de la faculté qu'a le muscle lisse au repos de maintenir une tension passive (Panner et Honig, 1967 ; Cooke et Fay, 1972). Un second aspect morphologique important au plan fonctionnel est qu'il existe, en proportion variable selon l'organe, l'espèce animale et la couche musculaire considérée (Gonella, Condamin et Roman, 1975), des zones de juxtaposition ou de contact entre cellules musculaires lisses adjacentes. Ces zones (gap-junctions, nexus) permettent, par leur faible résistance électrique, la conduction de l'excitation d'une cellule à l'autre (Daniel et al., 1971). Elles sont responsables du fait qua le muscle lisse du tube digestif se comporte comme un syncitium. En fait, la véritable unité fonctionnelle est le faisceau de fibres lisses (Gonella, 1978). Mais, par le biais des digitations qui unissent des faisceaux voisins, c'est une vériłable continuité électrique qui est assurée au sein d'une même couche musculaire.

Au plan fonctionnel, ce sont des mouvements ioniques qui sont responsables de l'activité électrique de la cellule musculaire lisse, et de l'activation mécanique des macromolécules protéiques intracellulaires. La nature des ions ef les mécanismes en cause ont fait l'objet de travaux de synthèse (Holman, 1968 ; Kuriyama, 1968 ; Siegman, 1975 ; El Sharkawy et Daniel, 1975 ; Marechal et Casteels, 1976). L'activation électrique de la membrane cellulaire résulterait essentiellement du mouvement des ions sodium et calcium (flux entrant dans la cellule). Les ions calcium sont directement responsables de l'activation de la machinerie contractile. 


\section{2. - Les différentes composantes de l'activité électrique du muscle lisse gastro-intestinal.}

Les propriétés mécaniques les plus caractéristiques de l'intestin, telles que les exprimait Thomas (1955) sont la rythmicité et la polarité qui fait que les contractions tendent à se propager en direction aborale. Mais ces propriétés sont la traduction mécanique des phénomènes électriques générés au niveau des couches musculaires (Bortoff, 1975).

a) Rappel de quelques données électrophysiologiques.

Il existe entre les deux faces de la membrane de la cellule musculaire lisse au repos une différence de potentiel dite potentiel de repos (PR). Ce potentiel a fait l'objet d'estimations assez largement variables, en raison des difficultés rencontrées pour sa mesure ; il est dans la plupart des cas de 60 à $70 \mathrm{mV}$, l'intérieur de la cellule étant négatif par rapport à l'extérieur (Holman, 1968).

Lorsque la cellule musculaire lisse est excitée, sa membrane est le siège d'un potentiel d'action (PA) encore appelé potentiel de pointe (spike des auteurs anglo-saxons). Celui-ci est défini par Holman (1968) comme la réponse à une dépolarisation initiale, réponse au cours de laquelle la membrane se dépolarise un peu plus, et même acquiert une polarisation inverse de celle observée au repos. Puis le potentiel de la membrane revient au niveau de repos plus ou moins rapidement. Une grande variété de cas de figures autour de ce principe de base est rencontrée selon l'espèce animale et le territoire digestif considéré. On doit à Gonella (1978) d'en avoir fourni une systématisation simplifiée : deux types de muscles lisses peuvent être distingués selon que le potentiel de la membrane fait ou non l'objet d'oscillations permanentes.

Le fundus gastrique, l'intestin grêle de Cobaye, le côlon distal du Lapin ef le côlon du Singe sont constitués de muscles lisses dont le potentiel de membrane est stable. Leur fonctionnement est relativement mal connu. Nous ne ferons qu'évoquer ultérieurement l'activité du fundus.

Parmi les muscles lisses dont le potentiel de membrane est soumis à des oscillotions permanentes, certains tels ceux du corps et de l'antre gastrique des Carnivores, du Porc, du Singe et de l'Homme produisent des PA dits « à plateau », à raison de 3 à 5 par $\mathrm{mn}$, chacun durant 5 à $6 \mathrm{sec}$; d'autres, tels les muscles de l'antre gastrique du Lapin, du Rat et du Cobaye, ainsi que les muscles lisses de l'intestin grêle de toutes les espèces (cobaye excepté) produisent des ondes lentes rythmiques en permanence.

\section{b) Activité électrique et motricifé gastrique.}

La description des potentiels à plateau, telle qu'elle résulte d'un enregistrement par micro-électrode intracellulaire (Daniel et Irwin, 1968 ; Gonella, 1978) peut être la suivante : une dépolarisation rapide suivie de repolarisation rapide constitue le potentiel initial que l'on observe à l'état pur sur l'estomac quiescent. Lorsque l'estomac se contracte, la repolarisation du potentiel initial n'est que partielle et suivie d'un second potentiel ou « plateau » à décours très lent et comportant selon les cas une repolarisation progressive ou une lente dépolarisation suivie de la repolarisation. L'amplitude 
ef la durée du plateau sont directement corrélées à la force de la contraction gastrique (Miolan et Roman, 1971, Szurszewskj, 1975). Enfin, lorsque son amplitude est importante, le plateau peut être surchargé d'oscillations rapides. Des images analogues peuvent être recueillies par électrode extracellulaire, en dérivation monopolaire et amplification à courant continu, par exemple chez le Porc (fig. 1, ligne 1) ou chez le Chien (fig. 2, ligne 2). En d'autres termes, les oscillations permanentes du muscle lisse antral comportent à la fois des composantes rapides (potentiel initial et éventuellement oscillations rapides surchargeant le plateau), et une composante lente (le plateau luimême). Cette description générale paraît valable aussi bien pour l'activité de la musculeuse longitudinale que pour celle de la musculeuse circulaire comme l'a montré Szurszewski (1973) par la technique du pont de saccharose.

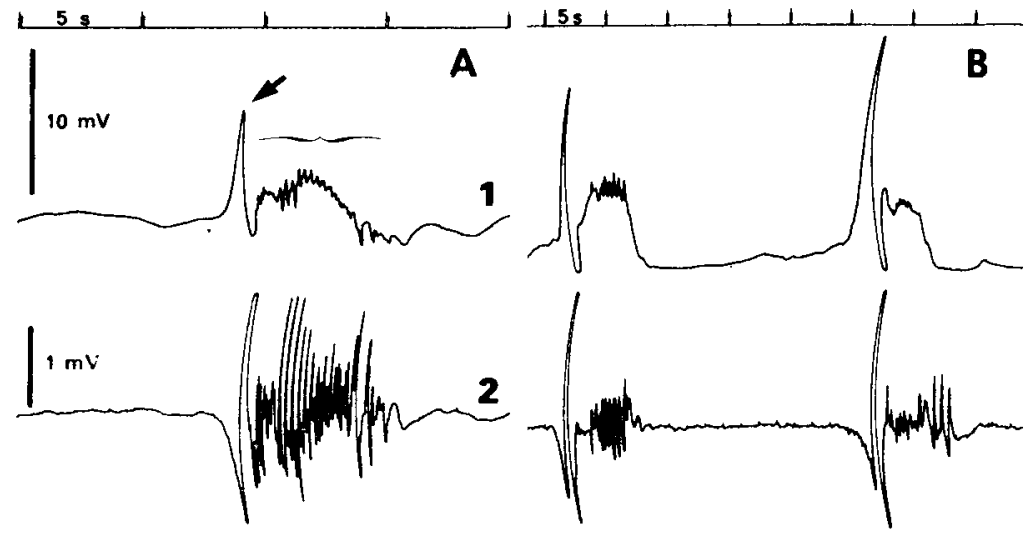

FIG. 1. - Electromyogramme de l'antre prépylorique chez le Porc en préparation chronique (d'après Laplace, données non publiées) :

Ligne 1 : enregistrement en méthode monopolaire, et amplification à courant continu. La flèche indique l'activité de contrôle (ECA). On note la forme variable de l'activifé de réponse (ERA) soulignée par l'accolade.

Ligne 2 : enregistrement en méthode bipolaire avec une constante de temps très faible $(0,03 \mathrm{~s})$.

L'électrode active en (1) est l'une des 2 électrodes réceptrices en (2). Celles-ci sont situées à $3 \mathrm{~cm}$ en amont du pylore.

Chez l'animal éveillé porteur d'électrodes implantées chroniques, il est possible d'enregistrer l'électromyogramme global de l'antre pendant de longues périodes de temps. Avec ce type de préparation, on utilise en général une amplification à constantes de temps très brèves, si bien que seules subsistent sur les tracés recueillis les composantes rapides du potentiel gastrique, à savoir le potentiel initial (dépolarisation-repolarisation rapides) et éventuellement les oscillations rapides qui se greffent parfois sur le plateau. Cette profonde différence d'aspect des tracés recueillis à partir des mêmes électrodes, selon les conditions d'amplification et d'enregistrement mises en œuvre, peut être constatée par la comparaison des lignes 1 et 2 des figures 1 (Porc) et 2 (Chien). La similitude d'aspect d'un tel électromyogramme chronique (au paramètre de temps près) avec un électromyogramme intestinal conduit bien souvent à appliquer la même 
terminologie (ondes lentes ef potentiels de pointe) dans les 2 cas, ce qui est en toute rigueur parfaitement faux.
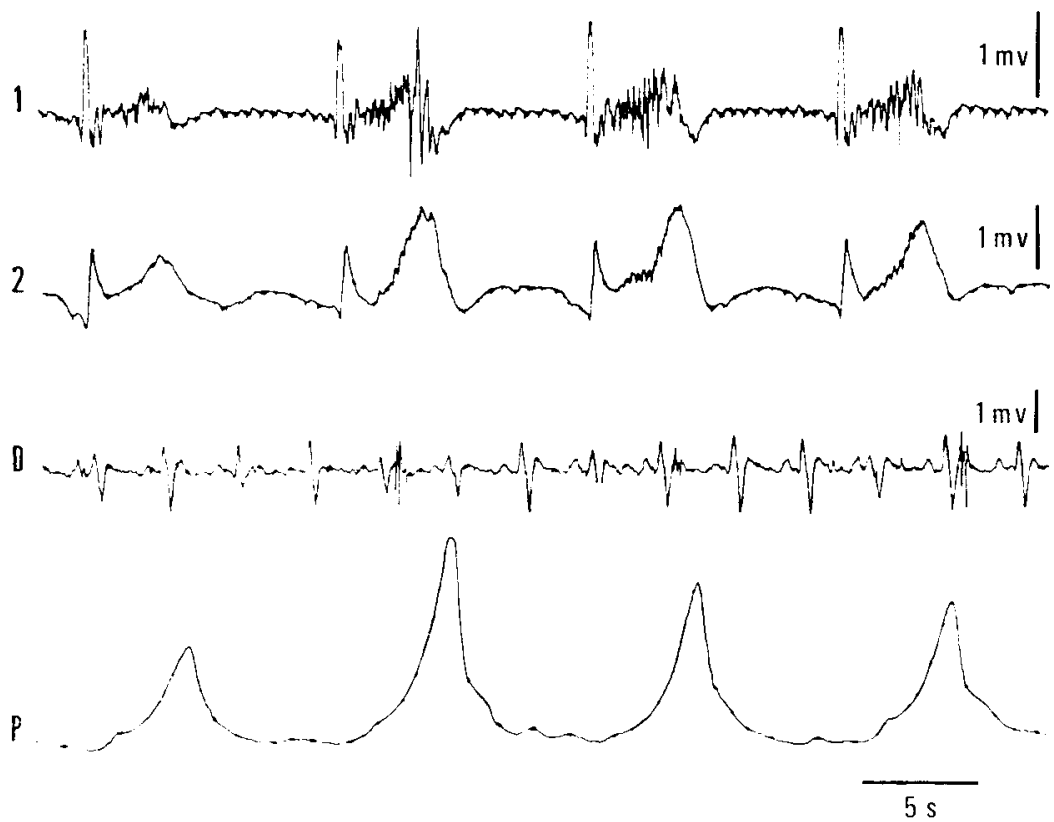

FIG. 2. - Electromyogramme de l'antre prépylorique chez le Chien en préparation chronique (d'après Miolan, 1974) :

1. Enregistrement en méthode bipolaire et faible constante de temps $(0,15 \mathrm{~s})$. Les électrodes réceptrices sont situées à environ $2 \mathrm{~cm}$ du pylore, l'axe interélectrode étant perpendiculaire au grand axe de l'estomac.

2. Enregistrement en méthode monopolaire et amplification à courant continu. L'électrode active est une des deux électrodes précédentes.

$D=$ Electromyogramme duodénal concomitant.

$\mathrm{P}=$ Pression intragastrique recueillie grâce à un cathéter à embout libre rempli d'eau, dont l'extrémité est située au niveau de l'antre pylorique.

L'important ici, en gardant à l'esprit le rôle de l'antre dans l'évacuation gastrique, est que c'est l'amplitude du plateau, effacé par l'électromyographie en constantes de temps brèves, qui conditionne la force de la contraction (fig. 2, lignes 2 et P). Celle-ci, lorsqu'elle apparaît, est toujours concomitante du " plateau ». Les oscillations rapides qui surchargent éventuellement ce plateau sont elles-mêmes concomitantes de la contraction, mais semble-t-il seulement lorsque cette contraction est forte, si bien qu'une contraction plus faible peut exister en leur absence. De fait, très marquées au niveau du pylore, ces oscillations sont d'autant plus réduites que l'on s'en éloigne en direction du corps. Par conséquent, les oscillations rapides qui sont le seul témoin électrique du plateau dans un électromyogramme chronique, et que l'on ne peut pas assimiler à des potentiels de pointe, constituent un témoin approximatif de la présence de contractions dans la zone antrale pré-pylorique, sans permettre une évaluation précise 

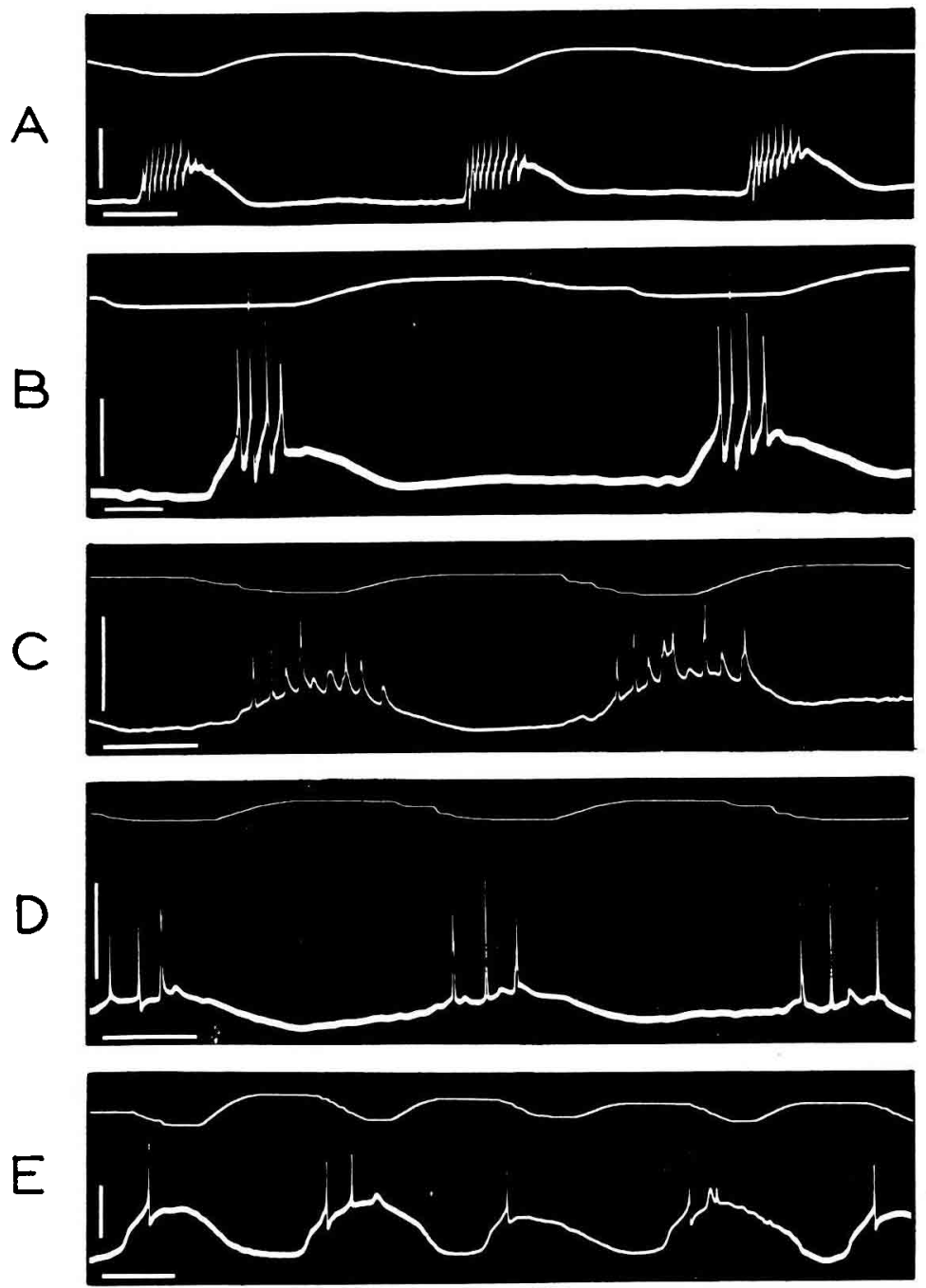

FIG. 3. - Enregistrements de l'activité électrique des fibres musculaires longitudinales du duodénum du Lapin in vitro (d'après Gonella, 1965).

Pour chaque enregistrement le tracé du haut représente le mécanogramme : une déflexion vers le haut traduit une contraction du segment intestinal ; le tracé du bas représente l'activité électrique dérivée par micro-électrode intracellulaire : une déflexion vers le haut traduit une positivation de la pointe de la microélectrode.

A ef B : Enregistrements obtenus à partir de deux préparations différentes dans la zone antimésentérique du duodénum.

$C$ et $D$ : Enregistrements effectués sur une même préparation en deux points différents de la zone mésentérique de l'ampoule duodénale.

$E$ : Même préparation qu'en $C$ et $D$ : dérivation de l'électromyogramme sur la zone antimésentérique de l'ampoule duodénale.

Tarages. - Amplitude : $20 \mathrm{mV}$. Temps : I s en A, C, D et E; 0,5 s en B. 
de leur force. En d'autres termes, s'il existe globalement une certaine proportionnalité entre l'importance des oscillations rapides et celle des activités mécaniques contemporaines (Monges, Salducci ef Roman, 1969), il n'y a pas relation causale et corrélation strictes entre ces deux ordres de phénomène.

\section{c) Activité électrique et motricité intestinale.}

Les oscillations permanentes du potentiel de membrane des muscles lisses de l'intestin grêle de toutes les espèces, à l'exception du Cobaye, sont des potentiels nettement différents de ceux observés au niveau gastrique. Il s'agit au niveau du grêle quiescent d'une variation lente de potentiel, plus connue sous le nom d'onde lente $(O L)$ qui peut exister à l'état pur et qui par elle-même n'est jamais associée à aucun phénomène mécanique. Ces OL ont fait l'objet de diverses descriptions (Burnstock, Holman et Prosser, 1963 ; Prosser et Bortoff, 1968 ; Bortoff, 1975). Quoique leur configuration varie selon l'espèce ou le segment d'intestin, il s'agit toujours d'un phénomène pseudosinusoïdal, chaque OL comportant une dépolarisation relativement rapide et soutenue, suivie d'une repolarisation graduelle. Lorsque la musculeuse intestinale se contracte, des potentiels de pointe (PA) se produisent, en surcharge de l'OL (fig. 3). Les OL surviennent rythmiquement à raison de 17 à 18 par mn au niveau du duodénum, et de 1112 par mn au niveau de l'iléon dans le cas du Porc par exemple. Leur fréquence d'apparition, systématiquement décroissante dans le sens oral-aboral, est une caractéristique du segment intestinal considéré, d'où la dénomination commune, appliquée aux OL, de rythme électrique de base (Basic electrical rhythm $=$ BER).
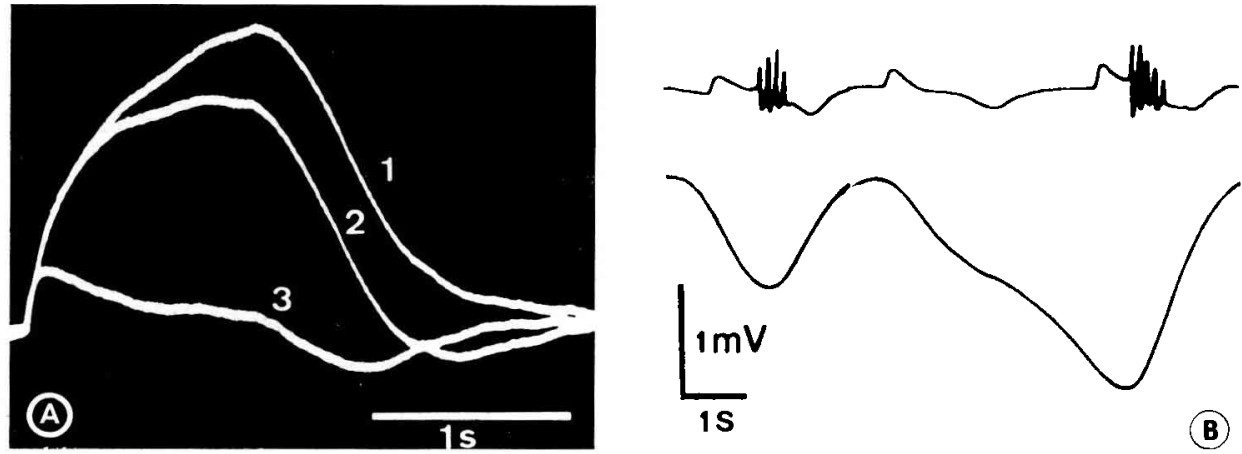

FIG. 4. - En A : Déformation d'une onde lente intestinale, due ò l'effet de filtre d'une amplification à liaison résistance-capacité (d'après Gonella), 1967).

Constance de temps du filtre : 1,5 s en (2) - 0,1 s en (3) - amplification à liaison continue en (1) Il s'agit dans les 3 cas de la même onde lente enregistrée préalablement sur bande magnétique avec amplificateur à courant continu. Enregistrement effectué in vivo.

En B : Relation entre activité électrique rapide (potentiels de pointe) et activité méconique de la musculeuse intestinale (d'après Gonella, 1967).

Tracé supérieur = électromyogramme dérivé par électrode extra-cellulaire, et enregistré avec une faible constante de temps $(0,1 \mathrm{~s})$.

Tracé inférieur = enregistrement isotonique des contractions. On observera sur l'EMG, entre les deux salves de potentiels de pointe, une onde lente à l'éfat pur. Elle correspond sur le myogramme à une absence de contraction d'où résulte un relâchement a normalement important du segment intestinal. 
En dépit du caractère généralement moins ponctuel des enregistrements mécaniques, il s'avère certain que les contractions intestinales sont suscitées par les PA et eux seuls (fig. 4). Ces potentiels ne survenant qu'en surcharge de l'OL, les contractions sont en étroite relation temporelle avec celle-ci. Notons enfin que la description rappelée ci-dessus de la forme des OL, et des PA éventuellement associés, repose sur l'image fournie lors d'enregistrement par micro-électrode intracellulaire. Comme dans le cas de l'estomac, l'enregistrement extracellulaire et plus encore l'électromyographie globale en constantes de temps brèves fournit une image différente du fait de la déformation des ondes lentes (fig. 4). Dans le cas de l'intestin grêle, cela reste sans conséquence méthodologique dans la mesure où la composante électrique rapide responsable de la contraction (PA) est préservée même avec une constante de temps très brève. Précisons enfin que les enregistrements recueillis au niveau de chacune des 2 couches musculaires intestinales ont sensiblement la même allure.

\section{3. - Origine et rôle des oscillations permanentes du potentiel de membrane.}

a) A propos de terminologie.

Afin de ne pas entretenir la confusion habituelle en matière de terminologie, nous utiliserons désormais dans le cas de l'estomac le terme proposé par Daniel et Irwin (1971) d'activité électrique de contrôle (Electrical control activity : ECA) pour désigner l'oscillation périodique du potentiel de membrane en l'absence de contraction, c'est-àdire le potentiel initial seul qui n'induit pas l'activation mécanique ; nous utiliserons le terme d'activité électrique de réponse (Electrical response activity =ERA) pour désigner le plateau qui provoque la contraction gastrique. Nous conserverons pour l'intestin les vocables usuels : ondes lentes (équivalent fonctionnel de l'ECA gastrique) et potentiels de pointe (homologue de l'ERA gastrique).

Cela étant, il est évident que les contractions, tant au niveau du muscle lisse gastrique qu'au niveau du muscle lisse intestinal, surviennent à un rythme au plus égal à celui des ECA ou des OL. Ces dernières sont donc à l'origine de la rythmicité inhérente des phénomènes mécaniques soulignée par Thomas (1955). Ce rôle de métronome à l'égard des contractions justifie l'usage courant du terme anglosaxon de potentiel « pacesetter » (marquant la cadence) pour désigner indifféremment les ECA ou les OL.

Nombreuses sont les fibres musculaires lisses de la paroi digestive dotées de cette propriété d'oscillateur, jouant le rôle d'entraîneur. L'oscillation générée au niveau d'une fibre se propage de proche en proche. On pourra se reporter à la revue de Bortoff (1976) pour une analyse détaillée du problème complexe de la genèse et de la propagation de l'OL. Néanmoins, la notion de propagation des OL doit être nuancée. Comme l'a discuté Gonella (1970), il est certain que cette propagation s'effectue bien par voie musculo-musculaire (nexus), mais aussi que la propagation d'une excitation - en réponse à une stimulation — ne s'effectue que sur une distance très restreinte. Les OL entrent théoriquement dans la catégorie des potentiels « locaux » c'est-à-dire faisant l'objet d'une propagation décrémentielle. Ceci signifie qu'il y a seulement transmission aux fibres adjacentes par diffusion électrotonique. Un très grand nombre de fibres de la couche longitudinale étant douées d'auto-activité, la propagation apparente traduit en réalité l'interaction de deux phénomènes : d'une part la conduction 
musculo-musculaire ; d'autre part l'auto-activité propre de chaque territoire musculaire. C'est-à-dire que deux territoires immédiatement voisins se comporteraient, s'ils étaient isolés électriquement l'un de l'autre, comme deux oscillateurs ayant leur rythme propre. Mais du fait de la conduction musculo-musculaire, l'oscillateur dont le rythme est le plus élevé va entraîner l'autre et ainsi de suite de proche en proche.

b) Muscle lisse gastrique.

Dans le cas de l'estomac, l'oscillateur dominant se trouve situé approximativement à mi-hauteur de la grande courbure, sans que l'on sache très exactement quelle est sa localisation tissulaire (couche longitudinale ou circulaire). Prosser et Bortoff (1968) font état de travaux montrant sur préparation d'estomac de chat que les ECA prennent origine dans la couche longitudinale. Mais nous avons précédemment signalé que la couche musculaire circulaire était par elle-même le siège d'ECA, comme en témoignent les en registrements de Szurszewski (1973). Quoi qu'il en soit, le pôle apical de l'estomac est totalement dépourvu d'activité électrique périodique. Les ECA générées par l'entraîneur dominant se propagent sur le corps et l'antre, imposant ainsi à toute cette partie aborale de l'estomac un même rythme d'ECA et partant un même rythme des ERA éventuellement associées. La propagation des ECA s'effectue à une vitesse croissante, depuis le corps gastrique $(0,5 \mathrm{~cm} / \mathrm{sec})$ jusqu'à l'antre $(2 \mathrm{~cm} / \mathrm{sec})$, ainsi que l'ont montré Daniel et Irwin (1968) chez le chien éveillé.

Les ERA, lorsqu'elles sont présentes, sont temporellement associées de façon étroite aux ECA. Il s'ensuit que les contractions se propagent elles aussi dans le même sens et à la même vitesse que les ECA. Encore faut-il souligner que les ERA n'accompagnent pas obligatoirement les ECA en chaque point de l'estomac : elles peuvent par exemple être présentes au niveau du corps et absentes au niveau de l'antre. Autrement dit, l'ERA ne serait pas un phénomène propagé à la manière des ECA ; il s'agirait selon Daniel et Irwin (1968) d'un phénomène sous contrôle local (contrôle nerveux notamment).

\section{c) Muscle lisse intestinal.}

On considère très généralement que les OL sont générées dans la couche musculaire longitudinale de l'intestin grêle. Cette conception soutenue par Connor, Prosser ef Weems (1974) est combattue par Taylor, Daniel et Tomita (1975). Selon ces derniers, les OL seraient générées par des cellules de la couche circulaire proches de la couche longitudinale. Quoi qu'il en soit, ces $O L$ sont enregistrées dans les 2 couches musculaires et on admet qu'il existe un couplage entre les deux musculeuses. La nature exacte de ce couplage est un sujet plus riche d'hypothèses et de controverses que de certitudes. Mais il reste certain que ce couplage existe et qu'il est sans doute responsable de la coordination de l'activité des 2 musculeuses. On sait par exemple que la contraction de la musculeuse circulaire, lorsqu'elle se développe, suit de très près celle de la musculeuse longitudinale. Cependant, toute contraction du muscle longitudinal n'est pas obligatoirement suivie de celle du muscle circulaire, comme cela devrait être le cas si le couplage des deux couches musculaires étaił d'origine purement myogène. Sans doute le système nerveux intramural intervient-il également dans ce couplage. Quelle qu'en soit la localisation précise, l'entraîneur dominant est situé au niveau 
du duodénum très proximal. Il ne domine cependant pas la totalité de l'intestin grêle dont la musculature lisse fonctionne en réalité comme une série d'oscillateurs couplés échelonnés sur la longueur du viscère. Il a été rappelé précédemment l'existence d'un gradient décroissant de la fréquence des $O L$ dans le sens oral-aboral. Cette décroissance est accompagnée d'une diminution parallèle de la vitesse de conduction apparente des $\mathrm{OL}$. Un tableau des fréquences ef vitesses enregistrées sur divers segments intestinaux de diverses espèces animales, selon les conditions expérimentales mises en œuvre, a été dressé par Prosser et Bortoff (1968).

La décroissance de la fréquence des $O L$ s'effectue par paliers. Ce fait, d'abord constaté sur la base des fréquences de contractions (Hasselbrack et Thomas, 1961) a été confirmé pour les OL elles-mêmes quelques années plus tard (Diamant et Borłoff, 1969). La longueur de chaque palier de fréquence est variable au long de l'intestin et chacun de ces paliers est séparé du suivant par une zone relativement courte $(1 \mathrm{à} 3 \mathrm{~cm})$ qui est le siège d'un phénomène de battement avec augmentation puis diminution périodique de l'amplitude des OL (waxing and waning $=W W$ des auteurs anglosaxons). Chaque palier paraît dominé par un entraîneur situé à son extrémité orale. La simulation d'un tel système (Diamant, Rose et Davison, 1970 ; Sarna, Daniel et Kingma, 1971) suggère que la fréquence d'un entraîneur peut être accrue par l'influence des oscillations du plateau plus proximal, d'où la notion première d'un entraîneur dominant duodénal. Il apparaît en fait que c'est par l'existence de cette organisation myogène qu'est assurée en définitive ce que Thomas (1955) considérait comme l'une des propriétés mécaniques essentielles de l'intestin : à savoir, sa polarité incitant à la propagation en direction aborale. Par ailleurs, le phénomène de WW (Prosser ef Bortoff, 1968), traduit simplement la succession de coincidence puis d'opposition de phase des oscillations émanant des 2 oscillateurs adjacents à la zone concernée. L'existence de ces zones de WW pourrait exercer, selon un très récent travail de Bortoff (1978), un certain rôle à l'égard de la propagation des contractions, avec facilitation en période de coïncidence de phase et bloquage en période de décalage de phase.

Ces diverses particularités des OL intestinales sont importantes. En effet, les $O L$ faisant l'objet d'une conduction et étant susceptibles d'augmenter la probabilité de décharge des PA sources de contraction, il paraît vraisemblable que le péristaltisme leur soit lié d'une certaine manière.

\section{d) Cas de la jonction gastro-duodénale.}

Il existe une coordination fonctionnelle entre l'estomac et le duodénum : en période d'évacuation gastrique, une contraction duodénale fait immédiatement suite à celle de l'antre. Au point de vue électromyographique, cela se traduit par le fait que les PA surchargent les seules $O L$ duodénales qui se produisent juste après une ECA avec ERA gastriques. Ce phénomène est observé aussi bien chez le Chien (fig. 5) que chez le Porc (Laplace, 1976).

La question se pose de savoir par quel mécanisme peut être assurée une telle coordination. Trois hypothèses peuvent être envisagées : a) mécanisme résultant de l'éjection à travers le pylore d'une certaine quantité de chyme qui provoque la contraction réflexe du duodénum proximal ; b) mécanisme faisant intervenir l'activité des plexus nerveux intramuraux ; $c$ ) mécanisme basé sur un phénomène myogène de conduction transpylorique de l'excitation. Cette dernière hypothèse serait la bonne 
selon Bortoff et Davis (1968) qui notent que les ECA peuvent franchir le pylore par diffusion électrotonique parvenant ainsi au duodénum. Les ECA augmenteraient la dépolarisation résultant des $O L$ duodénales, et renforceraient ainsi la probabilité de décharge de PA au niveau de la musculeuse intestinale.
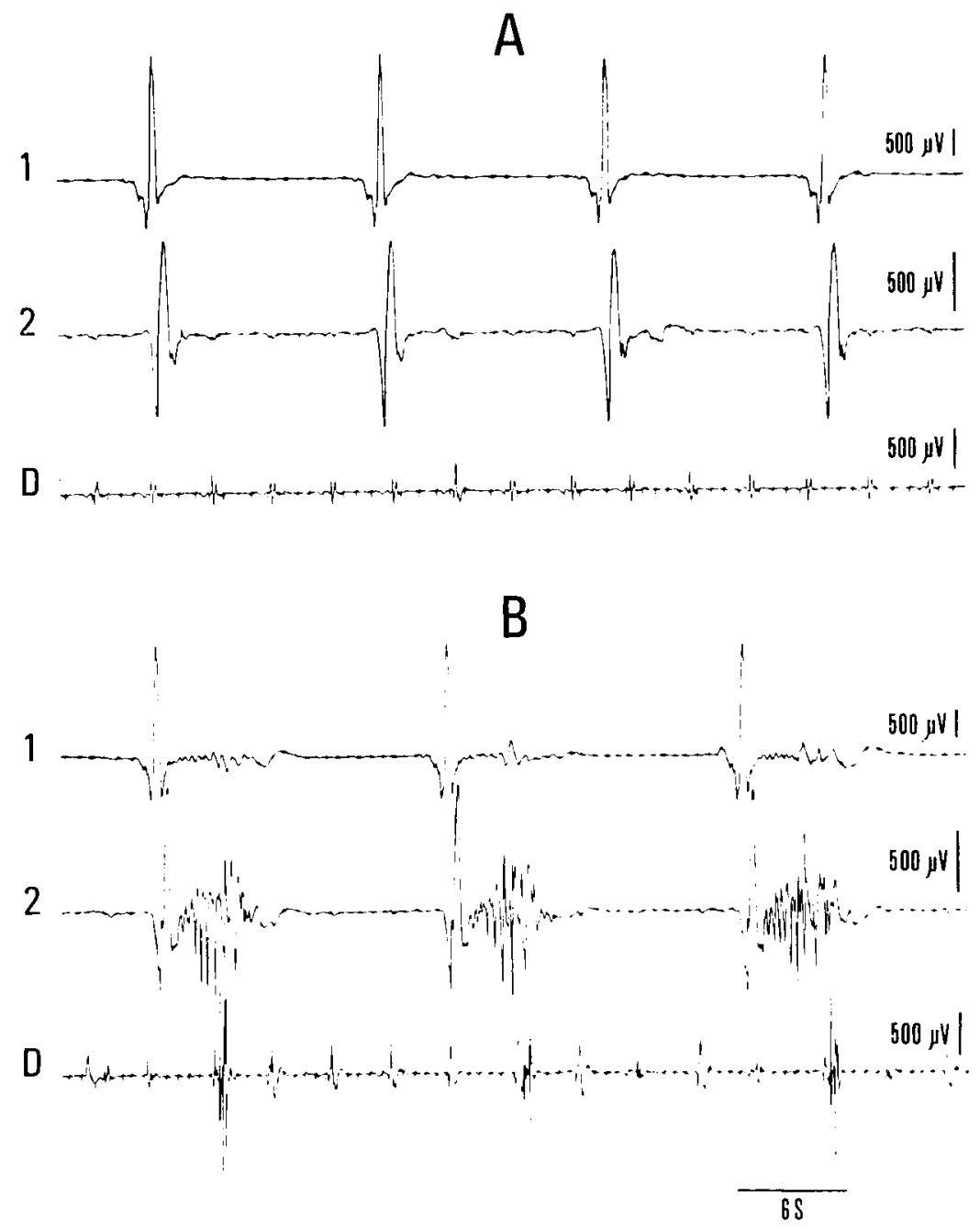

FIG. 5. - Eleciromyogramme gastroduodénal chez le Chien en préparation chronique, en $A$, chez le sujet à jeun depuis $24 \mathrm{~h}$, en $B, 1 \mathrm{mn}$ après ingestion de $400 \mathrm{~g}$ d'aliment complet (d'après Miolan, 1974).

1 et 2 = EMG antral enregistré en méthode bipolaire et faible constante de temps $(0,15 \mathrm{~s})$. Les électrodes 1 et 2 sont situées respectivement à environ $4 \mathrm{~cm}$ ef $2 \mathrm{~cm}$ en amont du pylore.

$D=E M G$ duodénal enregistré également en méthode bipolaire et faible constante de temps. Les électrodes sont situées sur une même génératrice transverse, environ $2 \mathrm{à} 3 \mathrm{~cm}$ en aval du pylore.

Noter en $B$ la chronologie des activiłés de réponse gastriques et des potentiels de pointe duodénaux qui indique l'existence d'un couplage fonctionnel entre les deux organes. 
Toutefois, les travaux de Atanassova $(1969 a, b, 1970 a, b)$ suggèrent que la coordination des activités électriques antrales et duodénales repose sur l'intégrité des plexus nerveux intra muraux, et que la propulsion de chyme gastrique joue un certain rôle dans la mise en œuvre de cette coordination. Selon cet auteur le phénomène myogène de conduction des ECA n'aurait pas un rôle décisif. Les conclusions d'Atanassova (1970b) quant au rôle du système nerveux intrinsèque semblent corroborées par les travaux de Bedi et Code (1972). Toutefois, ces derniers auteurs mettent en doute le rôle du passage du chyme dans le duodénum.

\section{4. - Organisation des phénomènes à l'échelle de l'organe.}

\section{a) Relation des activités des couches longitudinale ef circulaire.}

Pour une large part, nous avons jusqu'ici considéré ce qui se déroulait au niveau de la couche longitudinale. Il est néanmoins bien évident que le péristaltisme ne peuł s'exercer sans l'intervention combinée des 2 musculeuses. Il a été noté précédemment que les 2 couches musculaires gastriques présentent une activité électrique semblable, et que les enregistrements réalisés au niveau des 2 couches musculaires intestinales ont la même allure.

Cependant, il serait intéressant de pouvoir distinguer les activités suscitant l'activation mécanique (ERA ou PA selon le cas) en fonction de leur origine (musculeuse circulaire ou longitudinale) car, du fait même de leur disposition anatomique, les deux couches musculaires ont probablement des rôles différents. Cette distinction est malaisée. En ce qui concerne les ERA gastriques, aucune donnée n'est disponible. Dans le cas des activités rapides (PA) intestinales, les travaux de Gonella chez le Lapin (Gonella ef Gignoux, 1966 ; Gonella, 1967, 1970) ou de Ruckebusch (1972) chez le Cheval, apportent des éléments de distinction tout en confirmant par ailleurs que les OL ont effectivement pour origine la seule couche longitudinale. Ainsi, les PA de la musculeuse longitudinale (Gonella, 1970) sont généralement de faible amplitude ; au contraire, les PA de la musculeuse circulaire se présentent sous la forme de pointes de grande amplitude (Gonella ef Gignoux, 1966 ; Gonella, 1967). Parallèlement à ces informations recueillies in vitro, il semble bien que l'activité rapide du muscle circulaire constitue l'essentiel de l'électromyogramme global recueilli par des macroélectrodes implantées dans la paroi intestinale en préparation chronique. Selon Ruckebusch (1972) environ 90 p. 100 de l'activité rapide totale de la paroi, évaluée par intégration, seraient fournis par les PA de la musculeuse circulaire.

L'importance de la contribution du muscle circulaire dans l'électromyogramme total de la paroi ressort bien des observations de Bass (1968) réalisées à l'aide de jauges de contrainte disposées perpendiculairement de façon à en registrer séparément l'activité contractile de chacune des 2 couches : il existe une corrélation significative entre le nombre de PA par décharge, la durée des bouffées et la contraction du muscle circulaire. A l'inverse il ne semble pas y avoir de relation entre les PA et la contraction du muscle longitudinal. Ces faits sont confirmés par Oigaard et Dorph $(1974 a, b)$ qui soulignent la bonne corrélation entre la durée de la décharge de PAd'une part, et la durée et l'amplitude de l'onde de pression associée d'autre part, en dépit de la grande variabilité de l'amplitude des PA. 
En ce qui concerne la chronologie de l'activation des deux couches musculaires, il est clair qu'il existe en chaque point de l'intestin une précession de l'activité du muscle longitudinal sur celle du muscle circulaire (Gonella, 1967,1971). Encore faut-il rappeler un fait déjà évoqué à propos du couplage fonctionnel entre les deux couches, à savoir que chaque contraction de la couche longitudinale n'est pas forcément accompagnée d'une contraction de la couche circulaire. Enfin, Gonella (1972) a montré que l'activité du muscle longitudinal qui accompagne une $O L$ donnée est modifiée (réduction de la fréquence des PA, augmentation de la latence entre $O L$ ef PA) lorsqu'en ce même site l'OL précédente a été suivie d'une activation de la couche circulaire. La signification fonctionnelle de ce phénomène demeure encore obscure.

\section{b) Organisation myogène sous-jacente au péristaltisme.}

Nous avons noté précédemment que le péristaltisme devait être de quelque façon lié aux OL issues de la musculeuse longitudinale. De fait, il apparaît que le fonctionnement de la couche longifudinale fournit le support myogène d'un péristaltisme organisé. Mais c'est la couche circulaire qui, du fait de son « pilotage » par la longitudinale (couplage fonctionnel) et grâce à sa disposition anatomique permettant l'étranglement de la lumière, fait que le péristaltisme est efficace à l'égard des contenus. Ce support myogène est résumé par Bortoff (1976) de la façon suivante : le péristaltisme - c.-à-d. la contraction propagée impliquant les 2 couches musculaires - est la conséquence mécanique de la propagation aborale des OL, au long des paliers de fréquence ; l'amplitude d'une contraction péristaltique est une fonction du niveau global d'excitabilité dans la zone franchie par l'OL ; la distance parcourue par l'onde péristaltique n'excède généralement pas la longueur du palier de fréquence.

Cette formulation lapidaire représente l'aboutissement de divers travaux (Bortoff et Ghalib, 1972 ; Bortoff et Sacco, 1973 ; Bortoff, 1975) tendant à établir l'importance du rôle des OL : tant in vitro qu'in vivo la propagation de la seule OL, ne modifie pas le débit de sortie d'un segment intestinal perfusé, alors que la propagation d'une $O L$ surchargée de PA (ceux du muscle circulaire notamment) entraîne une augmentation de ce débit. Il faut préciser qu'à l'inverse de l'OL qui fait l'objet d'une propagation en direction aborale, les PA ne sont pas propagés (Bass, 1968). Ils sont suscités par l'OL qui passe en chaque site consécutivement, selon que les conditions locales le permettent ou non. Ainsi, une onde péristaltique n'est autre que la manifestation mécanique de la traînée de PA éventuellement suscités tout au long d'un territoire plus ou moins étendu par le passage d'une $O L$. De fait, l'OL et l'onde péristaltique se déplacent à une vitesse analogue d'environ 3 à $4 \mathrm{~cm} / \mathrm{sec}$ pour le duodénum de Lapin (Gonella, 1967, 1971 ) et de $2 \mathrm{~cm} / \mathrm{sec}$ pour le jéjunum de Chat (Bortoff, 1975).

Cependant, si important que paraisse ce rôle des $O L$, il faut en pondérer la portée. En effet l'OL peut très bien n'être suivie d'aucune activation mécanique des deux couches, ou que de la seule activation mécanique de la couche longitudinale. Donc, lorsqu'il y a péristaltisme c'est-à-dire propagation d'une activité coordonnée des deux couches, l'OL est effectivement nécessaire mais pas forcément suffisante. De fait, l'efficacité du travail propulsif d'un segment iléal isolé et perfusé est réelle, mais elle est 20 fois plus faible sous tétrodotoxine (Weems et Seygal, 1978) qui supprime l'intervention possible du système nerveux intramural. Ceci signifie que l'intestin est peut être 
capable de fonctionner sur une base myogène exclusive, mais que ce fonctionnement est beaucoup plus efficace lorsque le système nerveux intramural est actif.

c) Les contractions gastriques résultant de l'organisation des activités électriques.

La description des divers types de mouvements de l'estomac a été fournie par Code et Carlson (1968) et par Weisbrodt (1974). La classification proposée par Code et Carlson (1968) sur la base de données manométriques, distingue 3 types d'onde. On peut estimer que toutes trois sont de nature péristaltique. Cela est certain pour les ondes dites I et II qui se traduisent par des pics de pression isolés, survenant de façon rythmique dans l'estomoc actif, à la fréquence des ECA caractéristique de l'espèce animale considérée. Ces ondes I et II ne diffèrent que par leur amplitude qui est plus grande dans le cas des ondes II. Les ondes dites de type III ont été décrites comme des pics de pression groupés (train d'ondes péristaltiques) greffés sur une élévation de la pression basale. L'élévation de la pression basale a été reliée à un phénomène tonique sous-jacent. En réalité cette conception est discutable. Compte tenu de l'inertie de l'enregistrement manométrique, l'élévation de la pression basale pourrait n'être qu'une figure liée à la coalescence de la base des fortes ondes de pression ou encore résulter d'une sommation des pressions développées par les ondes péristaltiques qui affectent divers points de l'estomac, pressions qui sont transmises par les contenus (Weisbrodt, 1974).

Ces ondes contractiles naissent en général au niveau du corps de l'estomac. Leur propagation en direction du pylore concerne un territoire d'étendue variable. Un petit nombre seulement de ces ondes naît et s'achève sur le corps gastrique. Les plus vigoureuses parcourent en général l'antre en tout ou partie. Certaines s'éteignent progressivement en parcourant l'antre gastrique, et ne propulsent que fort peu de digesta vers le duodénum. D'autres franchissent la totalité de l'antre avec une vigueur croissante (à la mesure de l'épaississement de la musculature circulaire en direction aborale) et s'achèvent en une contraction quasi simultanée de l'antre terminal et du canal pylorique, véritable systole antrale. Ces contractions propulsent des quantités non négligeables de contenus, qui sont évacués dans le duodénum tant que le pylore demeure ouvert.

Le comportement que nous venons de décrire résulte du fait qu'au niveau gastrique, l'ECA a une fréquence uniforme, mais se propage à une vitesse croissante jusqu'au pylore. Lorsque l'ECA est accompagnée, sur tout ou partie de son trajet, d'une ERA avec activation conjuguée des 2 couches musculaires, la contraction contemporaine parcourt le même trajet, avec une vitesse croissante en direction aborale. La conjugaison de cette vitesse élevée dans la zone terminale avec une activation maximale de la couche circulaire est responsable des systoles antrales. L'étendue du territoire gastrique concerné par les ERA et l'intensité de ces derniers varient selon l'état d'excitabilité des couches musculaires, en fonction d'influences nerveuses et humorales multiples dont l'inventaire déborderait le cadre de notre sujet.

d) Les mouvements infestinaux résultant de l'organisation des activités électriques.

Au niveau de l'intestin grêle, les $O L$ ont une fréquence progressivement décroissante dans le sens oral-aboral. Leur vitesse de propagation décroît également dans le même sens. Comme l'ont exprimé Code, Szurszewski et Kelly (1971) les OL déterminent la fréquence maximum ainsi que la vitesse de propagation des contractions. 
A l'échelle d'un segment intestinal, selon que l'excitation affecte ou non la couche circulaire et selon que l'activité de celle-ci accompagne sur une plus ou moins grande distance l'activité de la couche longitudinale, trois types de mouvements de l'intestin peuvent être produits. On pourra se reporter pour leur description à la revue de Hightower (1968) ou à celle de Weisbrodt (1974). Notons seulement ici leur correspondance avec les activités électriques (Gonella, 1967, 1978) :

- Mouvements pendulaires : les OL sont surchargées des PA responsables de l'activation mécanique de la seule couche longitudinale.

- Mouvements de segmentation : aux phénomènes d'activation de la couche longitudinale s'ajoutent en certains points les PA de la couche circulaire, d'où une contraction circonférentielle localisée du viscère. Ce processus peut exister simultanément en plusieurs points d'un même segment intestinal. Celui-ci paraît alors marqué de constrictions plus ou moins régulièrement espacées sur toute sa longueur ; en outre, ces constrictions locales se produisent rythmiquement : leur fréquence maximum est celle des OL du segment intestinal considéré pour l'espèce concernée. Ce phénomène est connu sous le nom de segmentation rythmique.

- Mouvements péristaltiques : la contraction de la couche circulaire ne reste pas un phénomène strictement localisé, mais elle accompagne la contraction de la couche longitudinale sur une distance plus ou moins importante.

A cette classification traditionnelle des mouvements de l'intestin que l'on doit à Cannon (1902), on peut en substituer une autre distinguant les contractions impliquant la seule couche longitudinale, et les contractions mettant en jeu les 2 couches musculaires. Dans ce second groupe de contractions, une distinction supplémentaire pourra être faite selon que, en un point précis d'enregistrement, les bouffées de PA surviennent de façon rythmique (cas de la segmentation rythmique) pendant un certain temps, fournissant ainsi une activité de pointes régulière (Regular spiking activity $=\mathrm{RSA}$ ) ou que les bouffées de PA surviennent de façon aléatoire (cas d'une contraction locale isolée ou du passage d'une onde péristaltique quelle que soit la distance de sa propagation) fournissant une activité de pointes irrégulière (Irregular spiking activity = ISA). C'est sur la base d'une telle classification, qui semble prévaloir à l'heure actuelle, que nous allons aborder le rôle fonctionnel de ces divers types de contractions à l'égard du transit proprement dit, et en relation avec l'organisation spatio-temporelle de la motricité gastro-intestinale.

\section{5. - Organisation spatio-temporelle de la motricité gastro-intestinale.}

C'est Szurszewski (1969) qui a apporté la première description de l'existence d'une organisation spatio-temporelle des activités rapides (PA) de l'intestin grêle chez le Chien. Cet auteur a montré que l'intestin est périodiquement le siège d'une intense RSA, laquelle affecte au même moment tout un segment d'intestin et migre lentement dans le temps depuis le duodénum jusqu'à l'iléon. Cefte figure particulière est apparue (Code et Marlett, 1975) comme l'une des phases d'une séquence de phénomènes, soumise à récurrence cyclique et connue principalement sous le nom de complexe myoélectrique migrant (Migrating myoelectric complex $=M M C$ ). 
a) Complexes myoélectriques migrants de l'intestin grêle.

En un segment donné d'intestin, chaque MMC comporte trois phases principales consécutives (fig. 6). La phase dite I, dans la terminologie de Code et Marlett (1975), est caractérisée par l'absence quasi totale de PA, donc de contraction. La phase II ou phase d'ISA se distingue de la précédente par la présence de bouffées de PA, surchargeant très irrégulièrement et de façon apparemment aléatoire les $O L$; ces bouffées

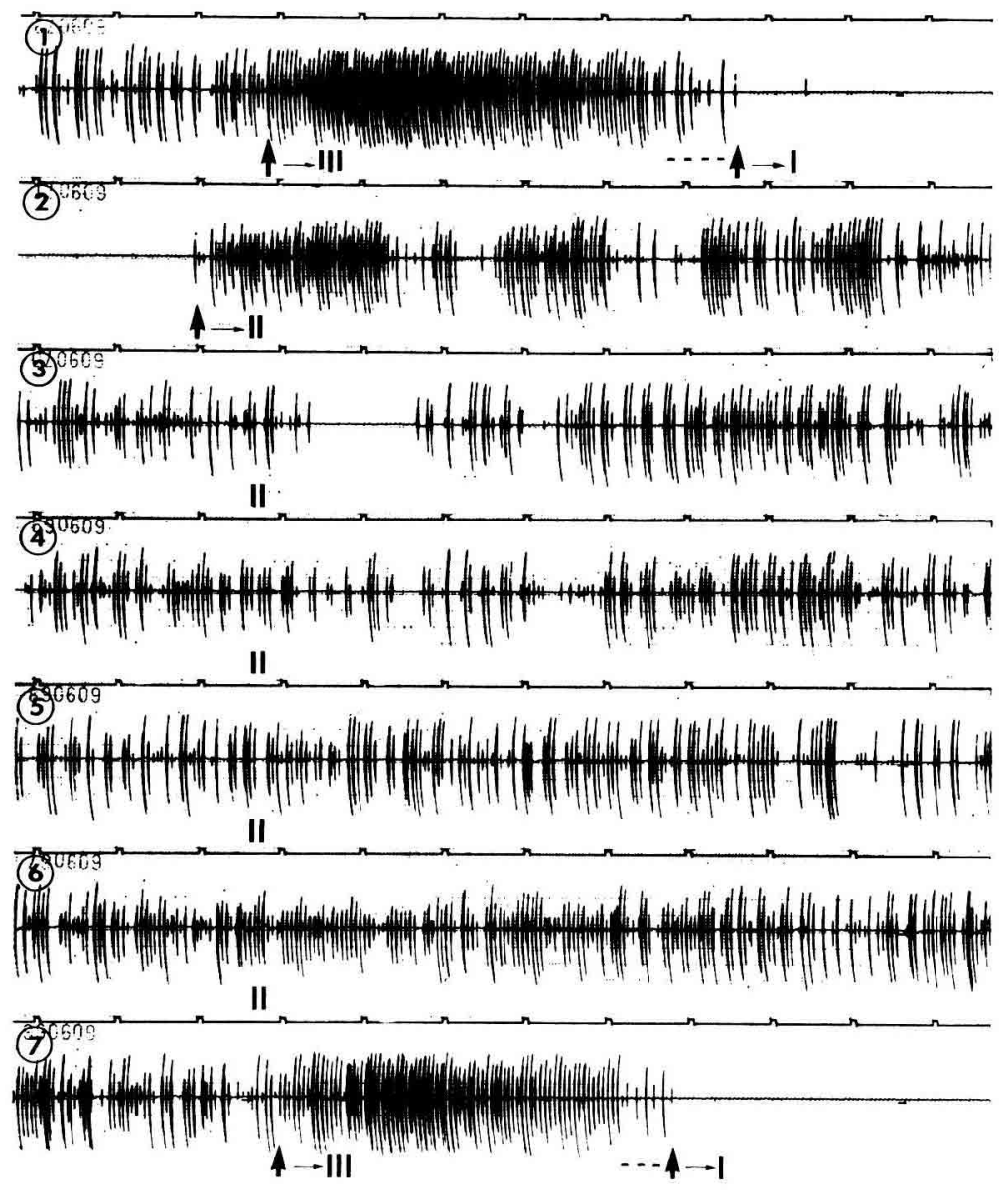

FIG. 6. - Electromyogramme duodénal global, dérivé à $16 \mathrm{~cm}$ du pylore chez le Porc et enregistré en méthode bipolaire et foible constante de temps $(0,03 \mathrm{~s})$. (d'après Laplace, 1978).

Les 7 bandes sont consécutives dans le temps, et illustrent le déroulement complet d'un complexe myoélectrique en un même site intestinal :

En 1, une phase d'activité de pointes régulière (phase III) dure $4 \mathrm{mn} 30$. Elle est suivie, après une période intermédiaire (pointillés) équivalant à la phase IV décrite par Code et Marlett (1975), par une période de quiescence (phase l) durant un peu plus de $5 \mathrm{mn}$. Puis on observe le déroulement jusqu'au début du tracé 7 , de la phase Il d'activité de pointes irrégulière. Enfin est enregistrée une nouvelle phase III $(4 \mathrm{mn})$ suivie de quiescence. La séquence complète du début de la phase I à la fin de la phase III dure $70 \mathrm{mn}$. La base de temps accompagnant chaque bande indique les minutes. 
correspondent à des fluctuations rapides, d'amplitude variable, de la pression endoluminale (Bueno, Fioramonti et Ruckebusch, 1975) ; les contractions, d'abord faibles et localisées, deviennent au fil du déroulement de la phase II de plus en plus intenses et de plus en plus souvent propagées (Code et Schlegel, 1973). Survient alors la phase III ou phase de RSA, phénomène initialement observé par Szurszewski (1969), et consistant en la surcharge systématique de chaque OL par une bouffée de PA; cette intense activité électrique (encore désignée sous le vocable de front d'activité, Szurszewski et Code, 1968 ; Code et Schlegel, 1973) correspond au plan mécanique à l'apparition de pics de pression surmontant une élévation de la pression basale (Bueno, Fioramonti ef Ruckebusch, 1975), c'est-à-dire à des phénomènes de segmentation rythmique décrits antérieurement sur la base d'enregistrements manométriques ou mécaniques sous le nom de burst of basic rhythm (Foulk et al., 1954) ou de burst pattern (Reinke, Rosenbaum et Bennett, 1967). Enfin un petit nombre d'auteurs identifie une phase IV qui correspond à un mélange irrégulier de contractions localisées et propagées (Code ef Schlegel, 1973). Cette phase peut être considérée comme la décroissance de la phase III et le prélude au silence moteur (Code et Marlett, 1975).

\section{b) Migration ef récurrence cyclique des MMC sur l'intestin grêle.}

La séquence ainsi'constituée fait toute entière l'objet d'une lente migration tout le long de l'intestin grêle et ce chez bon nombre d'espèces animales pour lesquelles des données ont été apportées au cours des dernières années. La vitesse moyenne de cette migration, calculée pour la partie proximale de l'intestin grêle, est variable selon l'espèce considérée : 3,5 à 11,7 cm/mn chez le Chien (Szurszewski, 1969 ; Carlson, Bedi et Code, 1972 ; Grivel et Ruckebusch, 1972 ; Code et Marlett, 1975); $10 \mathrm{~cm} / \mathrm{mn}$ chez le Lapin (Grivel ef Ruckebusch, 1972) ; $30 \mathrm{~cm} / \mathrm{mn}$ chez le Porc (Laplace, 1978) ; enfin $100 \mathrm{~cm} / \mathrm{mn}$ pour le Mouton (Grivel et Ruckebusch, 1972). De même au niveau de l'iléon, des vitesses comprises entre 1 et $40 \mathrm{~cm} / \mathrm{mn}$ peuvent être enregistrées selon l'espèce animale. Ces différences de vitesse de migration des MMC au long d'intestins grêles dont les longueurs varient dans un rapport de 1 à 10 selon l'espèce considérée, font que le temps de franchissement de tout l'intestin grêle par un MMC est dans tous les cas du même ordre de grandeur soit environ $120 \mathrm{mn}$ chez le Porc (Laplace, 1978), 97 à $120 \mathrm{mn}$ chez le Chien, 86 à $107 \mathrm{mn}$ chez le Lapin, 103 à $110 \mathrm{mn}$ chez le Mouton (Grivel et Ruckebusch, 1972).

Ce phénomène de migration continue du duodénum à l'iléon n'est toutefois pas absolument immuable. En effet, on constate chez le Porc (Laplace, 1978) que 40 p. 100 en moyenne des RSA ne franchissent pas tout l'intestin grêle. De même, 81 p. 100 (Szurszewski, 1969) ou les deux tiers (Grivel et Ruckebusch, 1972) des RSA seulement migrent jusqu'à l'iléon chez le Chien à jeun. Il en est de même chez le Mouton et le Lapin, pour lesquels les activités qui disparaissent ne franchissent en général que 40 p. 100 de la longueur de l'intestin, contre 60 à 70 p. 100 chez le Porc (Laplace, 1978), ef 60 ou 75 p. 100 chez le Chien (Grivel ef Ruckebusch, 1972 ; Szurszewski, 1969).

Ce fait mis à part, il existe d'une manière générale une grande stabilité de la récurrence cyclique des MMC. Ainsi chez le Chien, Szurszewski (1969) et Code et Marlett (1976) constatent que lorsqu'une RSA atteint l'iléon, une autre prend naissance ou est sur le point de se développer au niveau proximal. Ceci implique qu'existent chez le 
Chien au moins 1 et au plus $2 \mathrm{MMC}$ sur l'intestin grêle à un moment donné. Dans le cas du Porc (Laplace, 1978) ou du Veau (Dardillat et Marrero, 1977) ce sont par contre 2 ou $3 \mathrm{MMC}$ qui sont à tout instant présents en quelque point de l'intestin grêle.

De la succession dans le temps des différentes phases d'un MMC, et de leur migration dans l'espace, il résulte que si, à un instant précis, un segment donné est le siège d'une RSA (phase III), le territoire situé en amont est simultanément au repos (phase I), tandis que le territoire situé en aval fait simultanément l'objet d'une ISA (phase II). Cela signifie aussi qu'un segment intestinal, au repos à un certain moment, sera quelques instants plus tard atteint par une ISA, puis quelques dizaines de minutes plus tard par une RSA. Ces faits ressortent clairement de l'analyse de la figure 7, respectivement selon l'axe spatial (longueur du grêle) et selon l'axe des temps.

La principale différence interspécifique concernant le MMC est que cetłe organisation myoélectrique n'est visible que chez le sujet à jeun dans le cas du Chien (Szurszewski, 1969 ; Carlson, Bedi et Code, 1972 ; Code et Marlett, 1975) du Rat (Ruckebusch

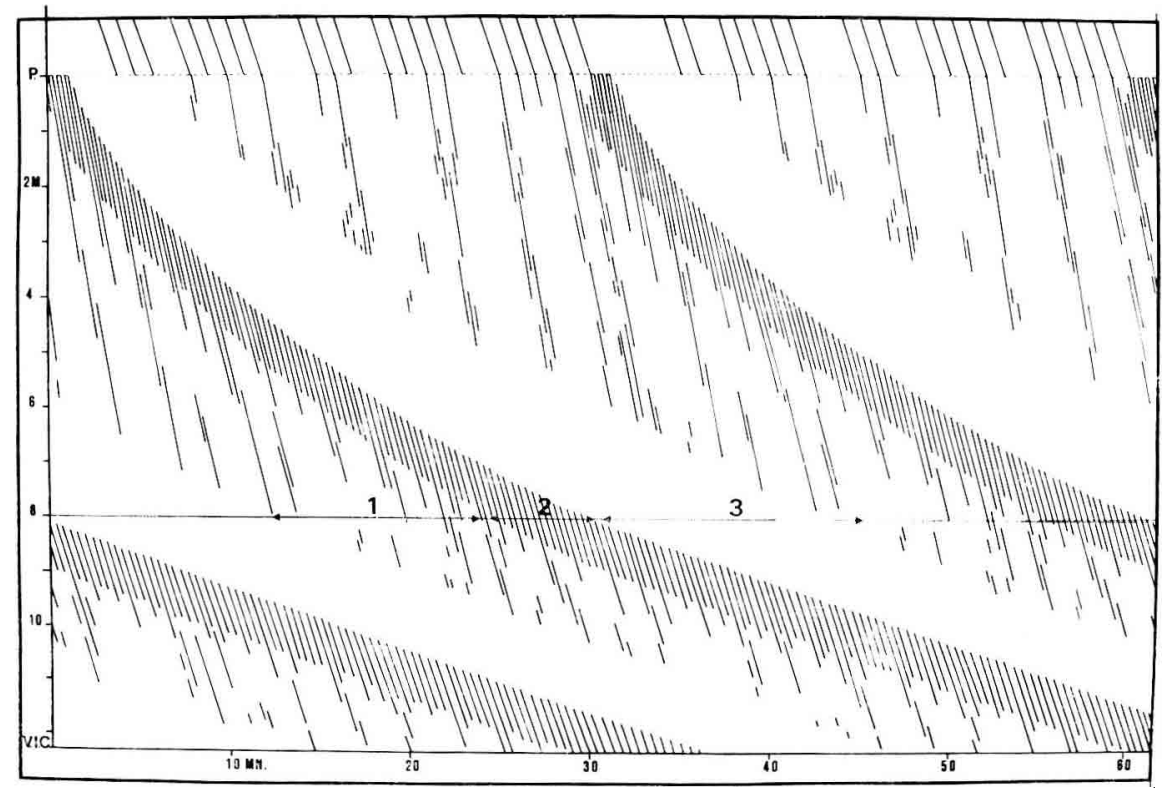

FIG. 7. - Représentation schématique de la propagation des salves de potentiels de pointe, et de la migration des phoses des complexes myoélectriques sur l'intestin grêle du Veau nouveau-né (d'après Dardillat, 1977). En ordonnée : distance en mètres du pylore ( $P$ ) à la valvule iléo caecale (VIC) ; en abscisse temps en $\mathrm{mn}$.

Schéma construit à partir de moyennes des mesures réalisées. La propagation des salves de potentiels de pointe est schématisée par des tirets ; leur fréquence réelle est quatre fois plus élevée que celle qui est représentée. Ce graphique fait abstraction des éventuelles anomalies de vitesse ou de sens de propagation des phénomènes.

Sur le jéjunum, à $8 \mathrm{~m}$ en aval du pylore, est indiquée la succession des différentes phases d'activité : (3) phase de repos ; (1) phase d'activité irrégulière ; (2) phase d'activité régulière. On constate qu'au même instant 2 ou 3 phases d'activité régulière sont présentes sur l'infestin grêle, et qu'en amont du segment affecté par une activité régulière, l'intestin est au repos; en aval, il est le siège d'une activité irrégulière. 
et Ferre, 1973) et de l'Homme (Hellemans ef al., 1977) ; dans ces trois espèces, la figure caractéristique des MMC cède la place à une ISA très fournie et continue durant plusieurs heures après le repas. Ce phénomène est désigné sous le nom d'organisation postprandiale. Par contre chez le Mouton et le Lapin (Grivel ef Ruckebusch, 1972) ainsi que chez le Veau (Ruckebusch, Dardillat et Hatey, 1972) l'organisation en MMC est constamment observable. Enfin le Porc occupe entre ces 2 groupes une position a mbiguë dans la mesure où les MMC sont présents en permanence chez l'animal alimenté ad libifum, mais cèdent la place durant $2 \mathrm{~h}$ à l'organisation postprandiale lorsque l'animal fait un petit nombre de repas relativement volumineux (Ruckebusch et Bueno, 1976 ; Laplace, 1978).

c) Complexes myoélectriques au niveau de l'estomac.

L'estomac est également concerné par cette organisation en MMC (Code et Marleff, 1975 ; Hinsdale, Cabot ef Kohatsu, 1977). Là encore, la phase I se caractérise par l'absence de contraction (c.-à-d. au plan électrique, ECA seule) et la phase II par des contractions (ECA $+E R A)$ survenant au hasard à raison d'au moins 1 par 3 à $5 \mathrm{mn}$; la phase III est celle de présence de fortes contractions régulières pendant en moyenne $18 \mathrm{mn}$; la phase IV, qui n'est pas toujours présente, est une brève période pendant
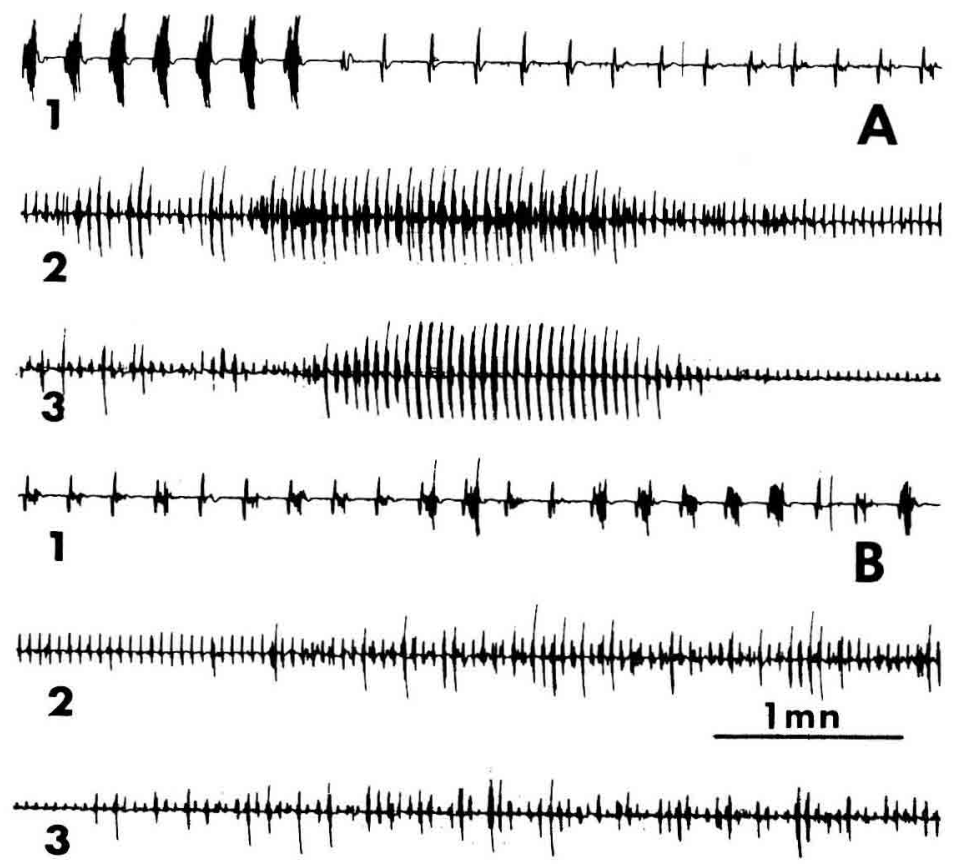

FIG. 8. - Electromyogromme (1) de l'antre pylorique d $2 \mathrm{~cm}$ du pylore, et (2 ef 3$)$ du duodénum respectivement à 13 ef $24 \mathrm{~cm}$ en aval du pylore chez le Porc (d'après Laplace, 1978).

Dérivations bipolaires. Constante de temps 0,03 s dans les 3 cas.

Les tracés B sont immédiatement consécutifs aux tracés homologues A. On note en A la naissance ef la propagation d'une phase d'activité régulière duodénale, concomitantes de la suspension des activités de réponse antrale. 
laquelle des contractions surviennent de façon erratique par groupes de 2 à 3 . Cette organisation dans le temps cède la place chez le Chien alimenté à une succession continue de contractions régulières. La séquence qui constitue le $M M C$ gastrique est interrompue par l'instillation de lait dans l'estomac ; la distension de celui-ci (Code ef Marlett, 1975) déclenche l'activité continue de type postprandial. Chez le Chien à jeun, Code et Marlett (1975) notent que estomac et duodénum sont soumis simultanément à la même phase du cycle dans 14 p. 100 des cas; dans tous les autres cas, il y a un décalage de $3 \mathrm{mn}$ seulement, l'estomac étant en avance sur le duodénum. Chez le Porc, le décalage est pratiquement constant ; en période postprandiale d'évacuation gastrique et de motricité antrale régulière continue, le démarrage sur le duodénum d'une RSA est contemporain (Ruckebusch et Bueno, 1976 ; Laplace, 1978) d'une suspension complète temporaire de toute activité antrale (fig. 8).

L'existence de ces complexes myoélectriques naissant à intervalles réguliers au niveau gastroduodénal et balayant lentement le tractus gastro-intestinal à une vitesse variable selon les espèces mais toujours progressivement décroissante dans le sens oral-aboral constitue, comme nous allons le voir, l'instrument d'une régulation du transit digestif à l'échelle de l'organe.

\section{6. - Motricité de l'estomac ef évacuation gastrique.}

\section{a) Rôle primordial de l'activité antrale propulsive.}

Comme cela a été évoqué précédemment, les contractions antrales jouent un rôle essentiel dans la réalisation de l'évacuation gastrique. Ce mécanisme a été maintes fois analysé ; il a été tout particulièrement bien décrit par Carlson, Code et Nelson (1966) grâce à l'emploi combiné chez le Chien des techniques cinéradiographiques, manométriques et électromyographiques. Nous empruntons à ces auteurs ce qui suit, qui est une synthèse de leurs diverses observations : une contraction annulaire relativement faible naît non loin du cardia, et se déplace en direction aborale sur le corps gastrique. Elle correspond au déplacement d'une ERA accompagnant la propagation d'une ECA. Lorsque cette contraction atteint l'incisura angularis de l'estomac, la constriction de la paroi s'approfondit, poussant du contenu du corps vers l'antre. Cette propulsion tend à distendre légèrement l'antre, en augmentant la pression endoluminale qui y règne. La résistance offerte par le canal pylorique (replis de muqueuse, tonus) étant surmontée, les contenus commencent à s'écouler dans le duodénum. La propagation de l'ECA s'effectuant à une vitesse apparente de plus en plus grande, la contraction qui l'accompagne franchit l'antre pour s'achever en une contraction quasi simultanée de l'antre terminal et du canal pylorique. Ce dernier se ferme donc, entraînant une brutale augmentation de la pression appliquée à la fraction non encore évacuée du contenu de l'antre terminal. Celui-ci est alors refoulé en direction de l'antre proximal voire du corps gasirique. Ce reflux forcé, dénommé rétropulsion, assure un brassage des digesta. Une autre démonstration de ce rôle des contractions antrales est apportée par l'implantation dans l'antre terminal d'un entraîneur électronique, qui conduit à inverser le sens de propa gation de l'ECA. Il y a alors un retard de l'évacuation des liquides et un ralentissement considérable de l'évacuation gastrique des solides (Sarna, Bowes et Daniel, 1976). 
Cela étant, bien que le péristaltisme gastrique soit le principal facteur de l'évacuation (motricité et évacuation sont abolies sous atropine) d'autres facteurs sont susceptibles d'intervenir. En effet, l'extrême simplicité apparente de la relation entre contraction antrale et évacuation gastrique reste parfois pleine de surprises au moins pour l'évacuation d'un repas d'épreuve liquide. Ainsi Stemper et Cooke (1975) notent qu'en général lorsque le nombre de contractions par mn (jauges de contrainte) augmente, le volume évacué par mn augmente lui aussi ; cependant un volume moyen de $6,9 \mathrm{ml}$ par mn peuf être évacué en l'absence de toute contraction antrale. Quoiqu'une évacuation gastrique rapide soit usuellement associée à l'augmentation de la force des contractions, des volumes de 0 à $67 \mathrm{ml}$ par mn peuvent être évacués en l'absence de contraction décelable. A l'inverse, des contractions puissantes n'aboutissent souvent qu'à l'évacuation d'un volume faible ou négligeable de contenus gastriques. Bien que Stemper et Cooke (1975) concluent à l'existence effective d'une relation entre activité contractile antrale et évacuation gastrique, il paraît clair que la motricité antrale n'est pas le seul facleur impliqué dans l'évacuation de l'estomac. D'ailleurs, ainsi que le souligne Weisbrodt (1974), l'atropine qui inhibe la motricité antrale, comme la morphine au pouvoir excito-moteur, conduisent toutes deux à une inhibition de l'évacuation gastrique. Notons, pour clore ce propos, que l'organisation dans le temps de la motricité antrale, liée à la présence de $M M C$, pourraił au moins chez le Porc, être responsable des irrégularités du décours de l'évacuation gastrique (Laplace ef Tomassone 1970).

b) Rôles du pylore et de la coordination gastro-duodénale.

Normalement le pylore est ouvert entre deux systoles antrales. II l'est cependant plus ou moins, et constitue en fait, en dehors de l'occlusion liée à la contraction antrale terminale, un «orifice à géométrie variable au niveau duquel s'exerce l'ajustement volumétrique des émissions gastriques » en fonction des pressions gastrique et duodénale (Laplace, 1972). Certaines hormones gastro-intestinales modifient ainsi tout à la fois motricité gastrique, ouverture de la lumière pylorique et évacuation gastrique. Par exemple, la cholécystokinine qui réduit l'activité gastrique et augmente la pression dans l'anneau pylorique (Fisher, Lipshułz et Cohen, 1973) inhibe (3 U/ kg/h) l'évacuation gastrique d'une solution saline (Debas, Faroog et Grossman, 1974). Les relations ne sont cependant pas toujours aussi claires. En effet, la gastrine accroît l'activité gastrique et abaisse la pression dans l'anneau pylorique (Fisher, Lipshutz et Cohen, 1973) mais ralentit $(0,1 \mu \mathrm{g} / \mathrm{kg} / \mathrm{mn}-\mathrm{IV}$ ) l'évacuation d'une solution saline (Dozois et Kelly, 1971). Quoi qu'il en soit, le rôle de la contraction antrale terminale (qui implique le pylore) est bien mis en évidence par le fait que l'antrectomie distale, qui supprime le sphincter pylorique (abolissant donc l'occlusion pylorique et la rétropulsion de digesta), accélère notablement l'évacuation des solides sans affecter sensiblement celle des liquides (Dozois, Kelly et Code, 1971). Le pylore jouerait donc un rôle de « barrière », sans doute plus important à l'égard des solides qu'à l'égard des liquides.

La coordination des activités gastriques et duodénales, déjà évoquée au paragraphe $3 d$, présente une importance non négligeable dans la mesure où, en période d'évacuation gastrique, la contraction duodénale se produit après la contraction antrale comme cela a été souvent observé à la suite de Thomas et Crider (1935). Ceci revient à dire que lorsque l'antre se contracte le duodénum est au repos, et que 
donc le mouvement des contenus s'effectue d'une zone de haute pression vers une zone de pression plus basse. Le bol évacué est ensuite pris en charge par le périsialtisme duodénal qui l'éloigne de l'estomac. Ce processus est assurément facilitateur de l'évacuation. Du reste, si l'on désorganise la motricité duodénale par implantation au niveau du duodénum distal d'un entraîneur électronique, l'inversion du sens de propagation des $\mathrm{OL}$ duodénales entraîne un reflux duodéno-gastrique important et un ralentissement de l'évacuation elle-même (Kelly et Code, 1977).

c) Influence du fundus dans le processus d'évacuation gastrique.

Le fundus gastrique, quoique dépourvu d'ECA (absence d'oscillations périodiques du potentiel de membrane des fibres de la musculeuse fundique), joue un rôle indirect dans l'évacuation gastrique, du fait que son activité peut modifier la pression intragastrique. On sait en effet depuis Cannon et Lieb (1911) que la partie orale de l'estomac présente un phénomène de relaxation lors de la déglutition. Cette relaxation se prolonge lors de déglutition répétitive, jusqu'à l'arrivée du dernier bol dégluti. Ce phénomène dit de « relaxation réceptive » permet de maintenir dans d'étroites limites la pression intragastrique lors de l'ingestion d'un repas. Cette relaxation serait due à une augmentation du potentiel de membrane des fibres fundiques (inhibition d'origine vagale). Inversement, une diminution du potentiel de membrane de ces mêmes fibres (excitation d'origine vagale) pourrait être à l'origine d'une tension soutenue (Szurszewski, 1977). Ces lentes modifications tensionnelles, qui font varier la pression endoluminale dans le fundus, et par contrecoup dans tout l'organe, seraient selon Kelly (1973) le facteur déterminant de la régulation de l'évacuation gastrique des liquides.

\section{7. - Le rôle des mouvements pendulaires intestinaux.}

La contraction de la couche circulaire étant toujours précédée de celle de la couche longitudinale, on peut considérer que le rôle essentiel des mouvements pendulaires est de déclencher l'activité de segmentation et de propulsion qui met en jeu la couche circulaire. Néanmoins, ces mouvements pendulaires pourraient avoir effectivement un rôle fonctionnel dans certains mouvements des contenus digestifs. C'est ce que suggère le travail de Melville, Macagno et Christensen (1975), quoique réalisé dans une situation in vitro sur un segment duodénal d'opossum et avec le recours à une modélisation simplifiée des mouvements de fluides (Macagno, Melville et Christensen, 1975). Il apparaît que la couche longitudinale est effectivement susceptible de se contracter isolément en l'absence de toute contraction circulaire, et que les déplacements longiłudinaux qui en résultent seraient susceptibles d'entraîner une circulation du fluide endoluminal. Celle-ci consisterait en un échange laminaire de fluide entre la veine centrale ef la périphérie de la lumière tubulaire intestinale.

\section{8. - Rôle des contractions intestinales résultant d'une activité coordonnée des deux couches musculaires.}

a) La relation motricité-transit.

Globalement, la relation entre les contractions de la musculeuse intestinale et le déplacement des contenus digestifs est vérifiée dans les situations de modification pro- 
voquée. Ainsi, Scott et Summers (1976) observent chez le Rat que le glucagon produit une inhibition des contractions (jauge de contrainte) jéjunales, et un ralentissement du transit, ces deux effets étant proportionnels à la dose utilisée (1-10-100 $\mu \mathrm{g} / \mathrm{kg} / \mathrm{mn})$. La caeruléine aux doses de 0,001 et $0,01 \mu \mathrm{g} / \mathrm{kg} / \mathrm{mn}$ entraîne une distribution plus uni-
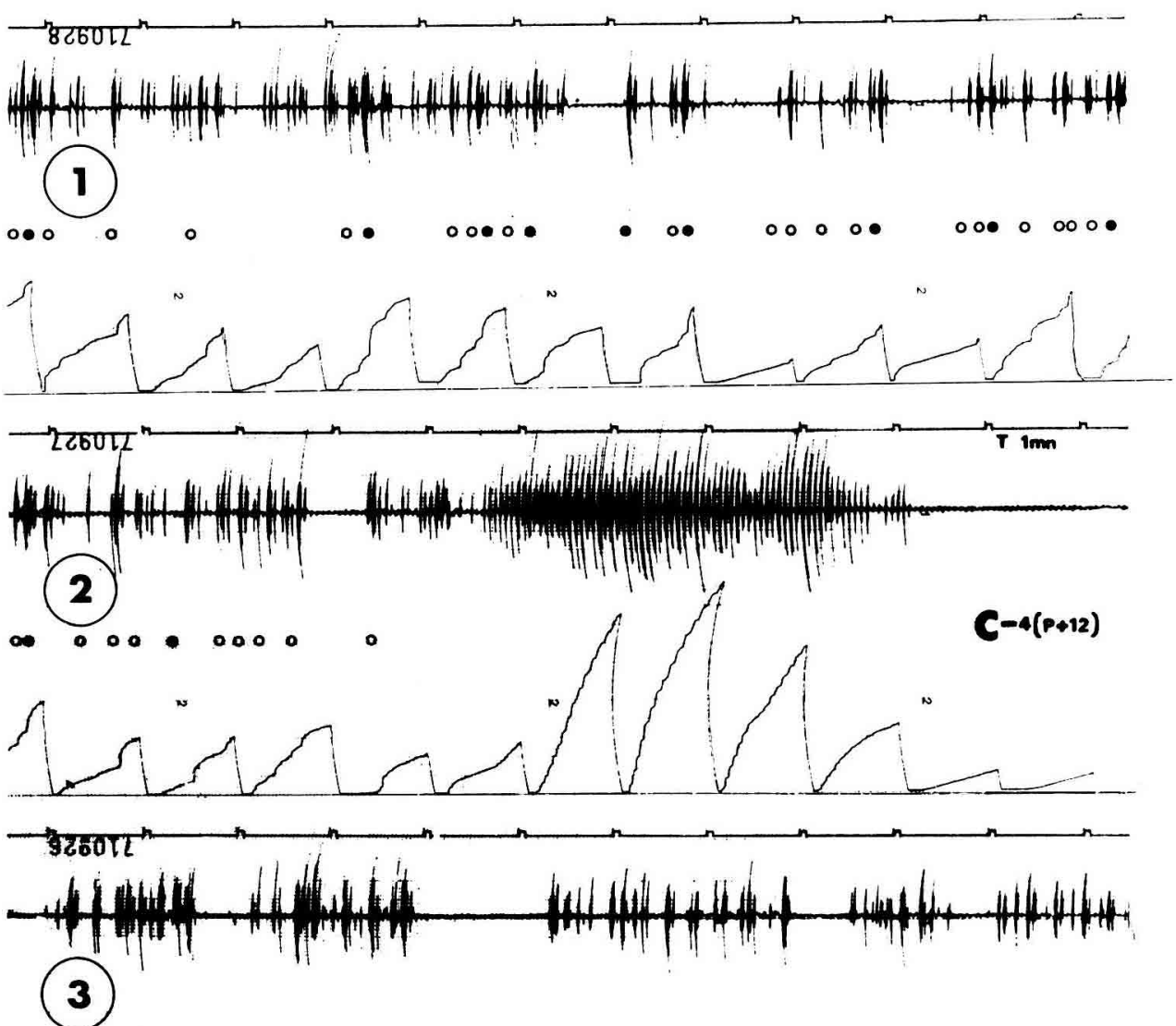

0.0000

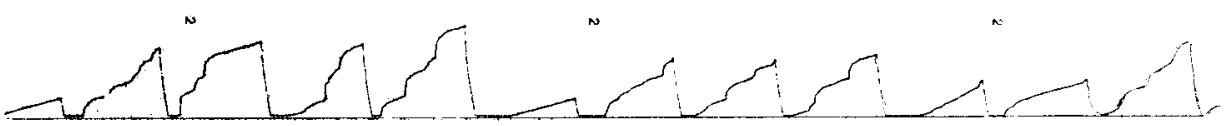

FIG. 9. - Relation chronologique entre les salves de potentiels de pointe au niveau du duodénum et l'écoulement des digesta au niveau d'une fistule réentrante chez le Porc (d'après Rérat, Corring et Laplace 1978).

La fistule est située à $12 \mathrm{~cm}$ en aval du pylore; l'électromyogramme est dérivé à $4 \mathrm{~cm}$ en amont de la canule proximale $(C-4(P+12)$.

Chaque bande 1-2-3 comporte successivement de haut en bas: la base de temps indiquant la minute ; l'électromyogramme duodénal (dérivation bipolaire-constante de temps $0,03 \mathrm{~s}$ ) ; la transcription des jets de digesta d'un volume inférieur à $10 \mathrm{ml}$ (cercles) ou compris entre 10 ef $30 \mathrm{ml}$ (points); l'intégration par unité de temps de $1 \mathrm{mn}$ des bouffées de potentiels de pointe duodénaux.

On notera que des écoulements de digesta ne sont observés que pendant la phase d'activité de pointes irrégulière. 
forme des contractions (motricité plus soutenue) et une accélération du transit ; à la dose de $1 \mu \mathrm{g} / \mathrm{kg} / \mathrm{mn}$ elle inhibe les contractions et retarde le transit.

La relation causale implicitement admise s'avérant globalement exacte, son analyse ultime devraił relier le déplacement d'une quantité donnée d'une certaine qualité de contenu avec la pression réelle développée dans la lumière par tel ou tel type de contraction. Une telle analyse s'avère cependant d'une extrême complexité, au moins lorsqu'elle est fondée sur les techniques manométriques (Tasaka et Farrar, 1976), du fait que toute pression endoluminale en un point est une résulfante de la force appliquée localement par la paroi sur les contenus et des pressions endoluminales régnant en amont et en aval. Quelques données plus précises ont cependant pu être obtenues récemment grâce à l'emploi de l'électromyographie combinée avec la manométrie, la débimétrie ou la cinéradiographie.

b) Complexes myoélectriques et transit dans l'intestin grêle.

Confrontant chez le Chien les données de l'électromyographie avec celles du radiocinéma, Code et Schlegel (1973) étaient arrivés à la conclusion que les MMC avaient un rôle propulsif. Mais du fait que chez le Chien les MMC ne sonf visibles qu'en période interdigestive, les auteurs leur avaient seulement attribué un rôle de nettoyage de l'intestin grêle pour le préparer à la réception du prochain repas. Ceci explique la dénomination de « housekeeper » alors proposée pour désigner les MMC. Comme on va le voir, les idées sur la signification fonctionnelle des MMC ont depuis lors évolué, en particulier au cours des 3 dernières années.

L'enregistrement simultané de l'électromyogramme et du débit intestinal (écoulement au niveau d'une fistule ou débimétrie électromagnétique) chez le Chien et le Mouton, a permis à Bueno, Fioramonti et Ruckebusch (1975) de constater que le passage des digesta survient pendant la période d'ISA (phase II). Au cours de cette période, il y a augmentation progressive du débit intestinal par augmentation des volumes déplacés et de la fréquence des passages. Par contre, au cours du déroulement de la période de RSA (phase III), aucun débit n'est observé ; les auteurs attribuent à cette activité un rôle de barrière de pression évitant tout reflux des digesta. Ces observations sont confirmées chez le Rat (Ruckebusch ef Fioramonti, 1975), le Veau (Dardillat, 1975), et le Porc (Rérat, Corring et Laplace, 1978) comme l'illustre la figure 9.

Summers, Helm et Christensen (1976) ont introduit dans le duodénum, à différents moments du déroulement d'un MMC chez le Chien, des bols de $2 \mathrm{ml}$ (solution isotonique de mannitol avec $100 \mu \mathrm{Ci}$ de $\mathrm{Na}_{2}{ }^{51} \mathrm{CrO}_{4}$ ). Ils observent que, lorsque l'injection a lieu pendant une ISA, il y a effectivement propulsion du bol en direction aborale. Si le bol est injecté au cours de la période de quiescence, il reste en place jusqu'à l'arrivée de I'ISA qui fait suite à la quiescence et c'est seulement alors qu'il sera propulsé en aval. Lorsqu'un MMC s'efface au cours de sa migration proximo-distale, comme cela se produit parfois, le bol reste dans le segment au niveau duquel s'est produit l'effacement; il n'est repris en charge que par I'ISA du MMC suivant.

Diverses informations présentées par Bueno, Fioramonti ef Ruckebusch (1975 ef 1977) suggèrent que le temps de passage dans l'intestin grêle est fonction des variations du nombre, de la durée ef de la vitesse de migration des MMC. Ces observations, fondées sur l'évaluation du débit de digesta au niveau proximal par référence à un marqueur de la phase liquide (polyéthylène glycol) perfusé à raison de $3 \mathrm{ml} / \mathrm{mn}$, suggè- 
rent aussi que les caractéristiques de la phase d'ISA des MMC sont directement fonction du volume des digesta. Plus précisément, il semblerait chez le Mouton que la durée de la phase d'ISA au sein d'un MMC varie au détriment ou au profit de la phase de quiescence selon qu'il y a respectivement surcharge ou déplétion de digesta (Ruckebusch et Bueno, 1977). Néanmoins, cette information ne peut à ce jour être tenue pour généralisable, d'autres modalités de réponse à des stimulations du type surcharge ou déplétion pouvant être observées (Laplace, observations non publiées). Au total, il nous faut souligner l'absence à l'heure actuelle de résultats expérimentaux établissant une relation précise entre le temps total de franchissement de l'intestin grêle par les matériaux alimentaires et les caractéristiques des MMC.

Dans un travail réalisé sur le Veau nouveau-né, et combinant électromyographie et débimétrie électromagnétique sur l'animal éveillé, Dardillat (1977) apporte un certain nombre de précisions sur le rôle propulsif des MMC. La quasi-totalité des débits de chyme est effectivement observée pendant la phase d'ISA. Aucun débit n'est observé au cours de la phase de RSA (fig. 10) ; cependant le front de progression de cette activité peut s'accompagner d'une ou plusieurs périodes de débit. Au sein de la phase d'ISA, seules sont accompagnées de débit les fortes salves de PA, propagées sur plusieurs centimètres et enregistrées successivement en amont puis en aval du débitmètre. Les débits semblent donc contemporains des fortes contractions péristaltiques. En un point déterminé, le débit commence bien avant que l'onde péristaltique arrive à ce niveau, et s'annule après son passage. Encore faut-il remarquer que beaucoup de bouffées de PA, enregistrées à proximiłé du débitmètre, ne s'accompagnent pas de débit. Cette observation est peut-être à rapprocher du fait que chez l'Homme (Oigaard et Dorph, 1974a, b) 20 p. 100 des salves de PA ne seraient accompagnées d'aucune

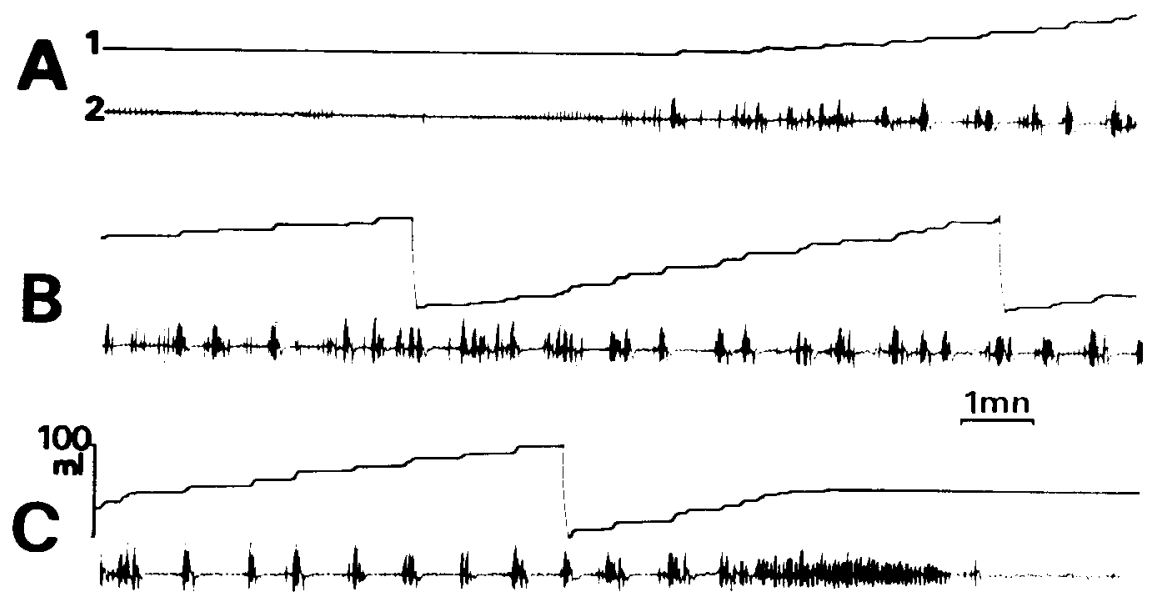

FIG. 10. - Evolution du débit, mesuré par débimétrie électromagnétique, dans le duodénum d'un Veau nouveau-né pendant le déroulement d'un complexe myoélectrique (d'après Dardillat, 1977).

En 1 : débits cumulés avec recyclage de l'intégrateur tous les $100 \mathrm{ml}$.

En 2 : électromyogramme duodénal au même site, soit à $20 \mathrm{~cm}$ du pylore.

Les tracés $A, B$ et $C$ sont consécutifs, et montrent successivement une phase de repos de 7 mn sans débit, une phase d'activité irrégulière de $29 \mathrm{mn}$ accompagnée de débits croissants en volume, une phase d'activité régulière de $3 \mathrm{mn}$ environ accompagnée d'un léger écoulement pendant le premier tiers de sa durée, puis à nouveau une phase de repos. 
onde de pression, et que seules les décharges d'une durée de plus de $3 \mathrm{sec}$ et d'une amplitude supérieure à $370 \mu \vee$ seraient assorties d'une onde de pression. Par ailleurs, selon les données de Dardillat (1977) le volume de chaque débit n'est pas directement proportionnel à l'amplitude ni à la densité de la bouffée de PA correspondant à l'onde péristaltique responsable du débit.

Le fait qu'en un site d'enregistrement, seules sont accompagnées d'un débit les fortes salves de PA, conduit cependant à s'interroger sur les limites de sensibilité du débitmètre électromagnétique. L'étalonnage qui en est fait fournit des réponses fidèles entre 10 ef $100 \mathrm{ml}$. La sensibilité en dessous du seuil de $10 \mathrm{ml}$ ne peut cependant être affirmée, à quoi s'ajoute une erreur de 5 p. 100 (Dardillat, 1977). Or on peut concevoir que des contractions naissant en amont du site d'enregistrement, ou que des contractions de faible puissance, ne poussent qu'un volume très faible de contenus, en deça du seuil de détection de l'appareillage. Cette observation, qui n'enlève rien à la valeur du travail de Dardillat (1977), nous paraît d'autant plus fondée que, chez le Porc par exemple, la mesure instantanée du volume de digesta s'écoulant au niveau d'une fistule fait apparaîfre le nombre très élevé de jets de faible puissance et d'un volume inférieur à $10 \mathrm{ml}$, voire d'écoulements de l'ordre du $\mathrm{ml}$ (Laplace, données non publiées). De la même façon il reste fort difficile de quantifier toutes les caractéristiques des bouffées de PA y compris l'aspect spatial de leur parcours. En somme, il n'est pas certain que les moyens d'investigation actuels soient suffisants pour permettre d'établir les corrélations très fines pouvant exister entre motricité et mouvements des contenus.

Ceci dit, il paraît se dégager de tous les travaux concernant le transit durant les MMC que, pendant les périodes de RSA, on n'observe pratiquement aucun transit, et ce bien que l'activité contractile de l'intestin soił alors très intense. La question se pose de savoir quelle peut être la signification fonctionnelle des RSA. Pour Dardillat (1977) les RSA auraient pour rôle de nettoyer le segment d'intestin concerné, en rassemblant les contenus étalés sur la surface de la muqueuse. Dardillat (1977) formule ainsi son interprétation du rôle respectif des ISA et RSA : « les bols de contenus, éjectés par les contractions antrales, sont rapidement poussés par les ondes propulsives duodénales (ISA) sur les premiers mètres de l'intestin. Ces bols disparaissent apparemment, soit par absorption, soit par dépôt sur les parois qui représentent une énorme surface. Ils n'atteignent pas le segment immédiatement aval qui est vide et qui est le siège d'une phase de repos. Ils se reconstituent au même niveau sur le front, ou en aval, d'une RSA, ce qui peut s'expliquer si l'on attribue un rôle de balayage à ces activités. Ils sont propulsés plus loin, puis disparaissent jusqu'à ce qu'ils soient à nouveau rassemblés par la phase de RSA suivante ».

Une autre interprétation, qui a priori n'est pas totalement contradictoire de la précédente, est que les RSA correspondent à de la segmentation rythmique. Cannon (1902), grâce à des observations radiologiques, a bien décrit ce phénomène chez le chat : " A string-like mass of food is seen lying quietly in one of the intestinal loops. Suddenly an undefined activity appears in the mass, and a moment later, constrictions at regular intervals along its length cut it into small ovoid pieces... ». Cette masse alimentaire, dans la description de Cannon (1902) est formée avant le déroulement de la segmentation rythmique ; elle n'est pas rassemblée par elle à partir de contenus étalés à la surface de la muqueuse.

Cette différence entre les observations de Cannon (1902) et l'interprétation de 
Dardillat (1977) provient peut-être du fait que le Chat, espèce utilisée par Cannon (1902) ne présente pas une organisation de la motricité intestinale en MMC aussi typique que chez les autres espèces. Par ailleurs, si l'on en croit Dardillat (1977) l'intestin serait vide en dehors des périodes d'ISA. En effet, l'aspiration à travers une canule ne fournit pas de digesta, ni pendant la phase de repos, ni pendant celle de RSA. Ce fait semble également en contradiction avec les observations de Cannon (1902) montrant une activité de brassage sur un segment intestinal qui est manifestement rempli, ne serait-ce que de substance radio-opaque. En fait cette dernière contradiction n'est peut-être pas réelle car il est possible que des essais de prélèvement par aspiration se révèlent inefficaces pendant la segmentation rythmique du fait de la compartimentation du contenu, due aux contractions stationnaires répétées. Finalement, si le rôle propulsif des ISA paraît bien établi, on peut encore s'interroger sur la signification fonctionnelle des RSA.

Nous concluerons sur cette dernière interrogation le cheminement qui nous a menés de l'activité électrique de la fibre musculaire lisse à la réalisation de la propulsion des contenus, en insistant sur le fait que la structuration myogène des activités gastro-intestinales constitue un « soubassement ». C'est sur cette base que s'exercent les influences nerveuses (systèmes nerveux intrinsèque ef extrinsèque) et humorales (hormones gastro-intestinales-sécrétions endocriniennes) grâce auxquelles le système est fonction nel et adaptable aux variations de l'alimentation.

\section{Commission CNERNA Digestion-Absorption/Association des Physiologistes, Poris 5-6 octobre 1978.}

\section{GLOSSAIRE}

- Termes utilisés avec indication de leur équivalent en anglais et éventuellement de l'abréviation employée dans le texte.

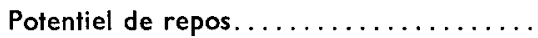

Potentiel d'action ou de pointe...........

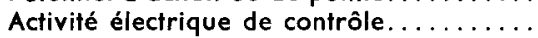

Activité électrique de réponse..........

Entraîneur . . . . . . . . . . . . . . .

Onde lente $\ldots \ldots \ldots \ldots \ldots \ldots \ldots \ldots \ldots \ldots$

Bouffée de pointes...............

Rythme électrique de base............

Phénomène de battement............

Activité de pointes régulière............

Activité de pointes irrégulière..........

Complexe myoélectrique migrant........

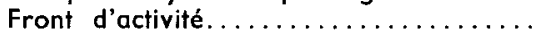

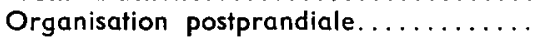

\section{- Synonymies :}

Potentiel initial gastrique. ............

Second potentiel gastrique............

Potentiel à plateau..................

Potentiel pacesetter. . . . . . . . . . . . .

Activité rapide. . . . . . . . . . . . . . . .

Activité lente..................

Complexe myoélectrique inter-digestif....

\begin{tabular}{|c|c|}
\hline 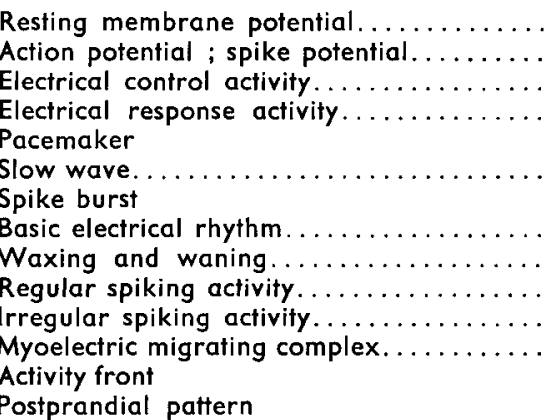 & $\begin{array}{l}\text { PR } \\
\text { PA } \\
\text { ECA } \\
\text { ERA } \\
\text { OL } \\
\text { BER } \\
\text { WW } \\
\text { RSA } \\
\text { ISA } \\
\text { MMC }\end{array}$ \\
\hline
\end{tabular}

$$
=\mathrm{ECA}
$$$$
=\text { ERA }
$$

Désigne l'ensemble ECA + ERA

Désigne indifféremment $O L$ ou ECA

Désigne les PA ou les oscillations des ERA gastriques Désigne les OL et les ECA gastriques

Se réfère au MMC pour les espèces où il n'est visible que chez le sujet à jeûn.

\section{- Homologies fonctionnelles :}

RSA : Segmentation rythmique, burst of basic rhythm, burst pattern.

ECA : OL

ERA : PA 


\section{Références}

ATANASSOVA E., 1969a. The role of the gastroduodenal junction in correlating the spike activities of the gastric and duodenal walls. C. R. Acad. bulg. Sci., 22, 947-949.

ATANASSOVA E., 1969b. The role of the intramural nervous system in correlating the spike activity between the stomach and the duodenum. C. R. Acad. bulg. Sci., 22, 1337-1340.

ATANASSOVA E., 1970a. Bioelectrical activity of the stomach and duodenum after cutting the gastroduodenal junction. Bull. Inst. Physiol. (Bulg.), 13, 211-227.

ATANASSOVA E., 1970b. On the mechanism of correlation between the spike activities of the stomach and duodenum. Bull. Inst. Physiol. (Bulg.), 13, 229-242.

BASS P., 1968. In vivo electrical activity of the small bowel, 2051-2074, chap. 100. In Handbook of physiology, Sect. 6, Alimentary Canal, Vol. 4 Motility. Amer. Physiol. Soc. Ed., Washington.

BEDI B. S., CODE C. F., 1972. Pathway of coordination of postprandial, antral and duodenal action potentials. Amer. J. Physiol., 222, 1295-1298.

BORTOFF A., 1975. Electrical activity of gastro-intestinal muscle, 17-29. In FRIEDMAN M. H. F., Functions of the stomach and intestine, Univ. Park Press, London.

BORTOFF A., 1976. Myogenic control of intestinal motility. Physiol. Rev., 56, 418-434.

BORTOFF A., 1978. Nature of the intestinal slow wave waxing and waning zone. Gastroenterology, 74, 1117 (abstr.).

BORTOFF A., DAVIS R. S., 1968. Myogenic transmission of antral slow waves across the gastroduodenal junction in situ. Am. J. Physiol., 215, 889-897.

BORTOFF A., GHALIB E., 1972. Temporal relationship between electrical and mechanical activity of longitudinal and circular muscle during intestinal peristalsis. Am. J. dig. Dis., 17, 317-325.

BORTOFF A., SACCO J., 1973. Myogenic control of intestinal peristalsis, 53-60. In Proc. 4th int. Symp. Gastro-intest. Motilify. Mitchell Press. Vancouver (Canada).

BUENO L., FIORAMONTI J., RUCKEBUSCH Y., 1975. Rate of flow of digesta and electrical activity of the small intestine in dogs and sheep. J. Physiol., Lond. 249, 69-85.

BUENO L., FIORAMONTI J., RUCKEBUSCH Y., 1977. Mechanisms of propulsion in the small intestine. Ann. Rech. vet., 8, 293-301.

BÜLBRING E., NEEDHAM D. M., 1973. A discussion on recent developments in vertebrate smooth muscle physiology, Phil. Trans. R. Soc. Lond. B, 265, 1-231.

BURNSTOCK G., HOLMAN M. E., PROSSER C. L., 1963. Electrophysiology of smooth muscle. Physiol. Rev., 43, 482-527.

CANNON W. B., 1902. The movements of the intestines studied by means of the Röntgen Rays. Am. J. Physiol., 6, 251-277.

CANNON W. B., LIEB C. W., 1911. The receptive relaxation of the stomach. Amer. J. Physiol., 29, 267-273.

CARLSON G. M., BEDI B. S., CODE C. F., 1972. Mechanism of propagation of intestinal interdigestive myoelectric complex. Am. J. Physiol., 222, 1027-1030.

CARLSON H. C., CODE C. F., NELSON R. A., 1966. Motor action of the canine gastroduodenal junction : A cineradiographic, pressure and electric study. Am. J. dig. Dis., 11, 155-172.

CODE C. F., CARLSON H. C., 1968. Motor activity of the stomach, 1903-1916, Chap. 93. In Handbook of physiology, Sect. 6, Alimentary canal, Vol. 4 Motility. Amer. Physiol. Soc. Ed., Washington.

CODE C. F., MARLETT J. A., 1975. The interdigestive myolectric complex of the stomach and small bowel of dogs. J. Physiol., Lond. 246, 289-309.

CODE C. F., SCHLEGEL J. F., 1973. The gastro-intestinal interdigestive housekeeper : Motor correlates of the interdigestive myoelectric complex of the dog, 631-634. Proc. 4th int. symp. Gastro-intest. Motility. Mitchell Press, Vancouver (Canada).

CODE C. F., SZURSZEWSKI J. H., KELLY K. A., 1971. A concept of motor control by the pacesetter potential in the stomach and small bowel. Am. J. Dig. Dis., 16, 601.

CONNOR J. A., PROSSER C. L., WEEMS W. A., 1974. A study of pacemaker activity in intestinal smooth muscle. J. Physiol., Lond., 240, 671-701.

COOKE P. H., FAY F., 1972. Correlation between fiber length, ultrastructure and the length-tension relationship of mammalian smooth muscle. J. Cell Biol., 52, 105-116. 
DANIEL E. E., IRWIN J., 1968. Electrical activity of gastric musculature, 1969-1984, Chap. 96. In Handbook of physiology, Sect. 6 Alimentary canal, Vol. 4 Motility, Amer. Physiol. Soc. Ed., Washington.

DANIEL E. E., IRWIN J., 1971. Electrical activity of the stomach and upper intestine. Am. J. dig. Dis., 16, 602-610.

DANIEL E. E., ROBINSON K., DUCHON G., HENDERSON R. M., 1971. The possible role of close contacts (nexuses) in the propagation of control electrical activity in the stomach and small intestine. Am. J. dig. Dis., 16, 611-622.

DARDILLAT C., 1975. Gastro-intestinal motility in calf neonatal disease, 111-122. In RUTTER J. M., Perinatal ill health in calves. Simpsons Press, Newburg (U. K.).

DARDILLAT C., 1977. Analyse électromyographique et débitmétrique du transit alimentaire chez le veau nouveau-né. J. Physiol. Paris, 73, 925-944.

DARDILLAT C., MARRERO E., 1977. Ełude de l'électromyogramme global chronique de la paroi intestinale du veau préruminant : migration des phases d'activité régulière et relation avec le transit. Ann. Biol. anim. Bicch. Biophys., 17, 523-530.

DEBAS H. T., FAROOQ O., GROSSMAN M. I., 1974. Inhibition of gastric emptying is a physiological action of cholecystokinin (CCK). Gasiroenterology, 66, 875 (abstr.).

DEWEY M. M., BARR L., 1968. Structure of vertebrate intestinal smooth muscle, 1629-1654, Chap. 81. In Handbook of physiology, Sect. 6 Alimentary Canal, Vol. 4 Motility, Amer. Physiol. Soc. Ed., Washington.

DIAMANT N. E., BORTOFF A., 1969. Nature of the intestinal slow wave frequency gradient. Am. J. Physiol., 216, 301-307.

DIAMANT N. E., ROSE P. K., DAVISON E. J., 1970. Computer simulation of intestinal slow-wave frequency gradient. Am. J. Physiol., 219, 1684-1690.

DOZOIS R. R., KELLY K. A., 1971. Effect of a gastrin pentapeptide on canine gastric emptying of liquids. Amer. J. Physiol., 221, 113-117.

DOZOIS R. R., KELLY K. A., CODE C. F., 1971. Effect of distal antrectomy on gastric empiying of liquids and solids. Gastroenterology, 61, 675-681.

EL SHARKAWY T., DANIEL E. E., 1975. Ionic mechanisms of intestinal electrical control activity. Amer J. Physiol., 229, 1287-1298.

FISHER R. S., LIPSHUTZ W., COHEN S., 1973. The hormonal regulation of pyloric sphincter function. J. clin. Invest., 52, 1289-1296.

FOULK W. T., CODE C. F., MORLOCK C. G., BARGEN J. A., 1954. A study of the motility patterns and the basic rhythm in the duodenum and upper part of the jejunum of human beings. Gastroenterology, 26, 601-611.

GONELLA J., 1965. Variation de l'activité électrique spontanée du duodénum de Lapin avec le lieu de dérivation. C. R. Acad. Sci., Paris, sér. D. 260, 5362-5365.

GONELLA J., 1967. Efude électrophysiologique de la motricité intestinole. Th. Doct. Sci Nat., Marseille.

GONELLA J., 1970. Etude de l'activité électrique de la couche musculaire longiłudinale du duodénum de lapin. J. Physiol., Paris, 62, 447-476.

GONELLA J., 1971. Etude électromyographique des contractions segmentaires et péristaltiques du duodénum de lapin. Pflügers Arch., 322, 217-234.

GONELLA J., 1972. Modifications of electrical activity of the rabbit duodenum longitudinal muscle after contractions of the circular muscle. Am. J. dig. Dis., 17, 327-332.

GONELLA J., 1978. La motricité digestive et sa régulation nerveuse. J. Physiol., Paris, 74, 131-140.

GONELLA J., CONDAMIN M., ROMAN C., 1975. Relation between the amount of nexuses in digestive smooth muscle and the amplitude of spikes recorded by extracellular electrodes, 152-157. In VANTRAPPEN G., AGG H. O., 5 th int. Symp. Gostrointest. Motility. Typoff-Press, Herentals (Belg.).

GONELLA J., GIGNOUX H., 1966. Ełude simultanée de l'activité électrique des fibres musculaires lisses longitudinales et circulaires du duodénum de Lapin in vitro. C. R. Acad. Sci., Paris, Série D, 262, 570-573.

GRIVEL M. L., RUCKEBUSCH Y., 1972. The propagation of segmental contractions along the small intestine. J. Physiol., Lond., 227, 611-625.

HASSELBRACK R., THOMAS J. E., 1961. Control of intestinal rhythmic contractions by a duodenal pacemaker. Am. J. Physiol., 201, 955-960. 
HELLEMANS J., VANTRAPPEN G., JANSSENS J., PEETERS T., 1977. Effect of feeding and of gastrin on the interdigestive myoelectrical complex in man, 29-31. Proc. 6th int. Symp. Gastrointest. Motility, MTP Press, Lancaster (Anglet.).

HIGHTOWER N. C., 1968. Motor action of the small bowel, 2001-2024. Chap. 98. In Handbook of physiology, Sect. 6 Alimentary canal, Vol, 4 Motility. Amer Physiol. Soc. Ed., Washington.

HINSDALE J., CABOT R., KOHATSU S., 1977. The migrating contractile complex of the stomach (abstr.). Gastroenterology, 72, 1069.

HOLMAN M. E., 1968. Introduction to electrophysiology of visceral smooth muscle, 1665-1708, Chap. 83. In. Handbook of physiology, Sect. 6 Alimentary Canal, vol. 4 Motility. Amer. Physiol. Soc. Ed., Washington.

HUDDART H., HUNT S., 1975. Visceral muscle. Its structure and function. Blackie, London, 181 pp.

KELLY K. A., 1973. Canine gastric motility and emptying : electric, neural, and hormonal controls, 463-470. Proc. 4th int. Symp. Gostrointest. Motility. Mitchell Press, Vancouver (Canada).

KELLY K. A., CODE C. F., 1977. Duodenal gastric reflux and slowed gastric emptying by electrical pacing of the canine duodenal pacesetter potential. Gastroenterology, 72, 429-433.

KURIYAMA H., 1968. Ionic basic of smooth muscle action potentials, 1767-1791. Chap. 87. In Handbook of physiology, Sect. 6 Alimentary Canal, Vol. 4 Motility. Amer. Physiol. Soc. Ed., Washington.

LAPLACE J. P., 1972. Motricité gastro-intestinale chez le porc : Etude descriptive par électromyographie et corrélations nutritionnelles. Rec. Med. vet., 148, 37-61.

LAPLACE J. P., 1976. L'excrétion biliaire chez le porc. 1) Electromyographie des voies biliaires extra-hépatiques. Rec. Med. vef., 152, 33-43.

LAPLACE J. P., 1978. Organisation de la motricité de l'intestin grêle chez le porc et influence de l'alimentation. Ann. Zootech., 27, 377-408.

LAPLACE J. P., TOMASSONE R., 1970. Evacuation gastro-duodénale chez le porc. Fistulation chronique par voie thoracique extra-pleurale. Recherche d'une technique d'analyse mathématique de l'évacuation. Ann. Zootech., 19, 303-332.

MACAGNO E., MELVILLE J., CHRISTENSEN J., 1975. A model for longitudinal motility of the small intestine. Biorheology, 12, 369-376.

MARECHAL G., CASTEELS R., 1976. Les muscles lisses de vertébré, 1715-1788, Chap. 21. In : KAYSER C., Physiologie, tome 2. Système nerveux, muscle. Flammarion, Paris.

MELVILLE J., MACAGNO E., CHRISTENSEN J., 1975. Longitudinal contractions in the duodenum : their fluid-mechanical function. Am. J. Physiol., 228, 1887-1892.

MIOLAN J. P., 1974. La motricité de l'estomac et du sphincter œesophagien inférieur : étude électromyographique ; rôle de l'innervation extrinsèque. Thèse Doct. Spécial. Physiol. anim., Univ. Aix-Marseille III.

MIOLAN J. P., ROMAN C., 1971. Modification de l'électromyogramme gastrique par stimulation des nerfs extrinsèques, J. Physiol., Paris, 63, 561-576.

MONGES H., SALDUCCI J., ROMAN C., 1969. Ełude électromyographique de la motricité gastrique chez l'homme normal. Arch. franc. Mal. App. Digest., 58, 517-530.

NEEDHAM D. M., SHOENBERG C. G., 1968. Proteins of the contractile mechanism in vertebrate smooth-muscle. 1793-1810, Chap. 88. In Handbook of physiology, Sect. 6 Alimentary Canal, vol. 4 Motility. Amer Physiol. Soc. Ed., Washington.

OIGAARD A., DORPH S., 1974a. The relative significance of electrical spike potentials and intraluminal pressure waves as quantitative indicators of motility. Am. J. dig. Dis., 19, 797-803.

OIGAARD A., DORPH S., 1974b. Quantitative analysis of motility recording in the human small intestine. Am. J. dig. Dis., 19, 804-810.

PANNER B. J., HONIG C., 1967. Filament ultrastructure and organization in vertebrate smooth muscle. Contraction hypothesis based on localization of actin and myosin. J. Cell Biol., 35, 303-321.

PROSSER C. L., BORTOFF A., 1968. Electrical activity of intestinal muscle under in vitro conditions, 2025-2050, Chap. 99. In Handbook of physiology, Sect. 6, Alimentary canal, Vol. 4 Motility. Amer. Physiol. Soc. Ed., Washington.

REINKE D. A., ROSENBAUM A. M., BENNETT D. R., 1967. Patterns of dog gastrointestinal contractile activity monitored in vivo with extraluminal force transducers. Am. J. dig. Dis., 12, 113-141. 
RÉRAT A., CORRING T., LAPLACE J. P., 1978. Quelques aspects des recherches en physiologie digestive chez le porc : applications possibles. Journées Rech. Porcine en France, Paris, INRAITP Ed., 10, 95-118.

ROZE C., COUTURIER D., DEBRAY C., 1976. Patterns of gastric electrical and motor activity in miniature pigs, Can. J. Physiol. Pharmacol., 54, 764-773.

RUCKEBUSCH Y., 1972. Origine de l'activité rapide de l'électromyogramme chronique de la paroi intestinale. C. R. Acad. Sci., Paris, Série D, 275, 1407-1410.

RUCKEBUSCH Y., BUENO L., 1976. The effect of feeding on the motility of the stomach and small intestine in the pig. Br. J. Nutr., 35, 397-405.

RUCKEBUSCH Y., BUENO L., 1977. Migrating myoelectrical complex of the small intestine. An intrinsic activity mediated by the vagus. Gastroenterology, 73, 1309-1314.

RUCKEBUSCH Y., DARDILLAT C., HATEY F., 1972. La motricité intestinale chez le veau nouveauné. Influence du repas. C. R. Soc. Biol., 166, 1547-1551.

RUCKEBUSCH M., FERRE J. P., 1973. Origine alimentaire des variations nycthémérales de l'activité électrique de l'intestin grêle chez le rat. C. R. Soc. Biol., 167, 2005-2009.

RUCKEBUSCH M., FIORAMONTI J., 1975. Electrical spiking activity and propulsion in small intestine in fed and fasted rats. Gastroenterology, 68, 1500-1508.

SARNA S. K., BOWES K. L., DANIEL E. E., 1976. Gastric pacemakers. Gastroenterology, 70, $226-231$.

SARNA S., DANIEL E. E., KINGMA Y. J., 1971. Simulation of slow wave electrical activity of small intestine. Am. J. Physiol, 221, 166-175.

SCOTT L. D., SUMMERS R. W., 1976. Correlation of contractions and transit in rat small intestine. Am. J. Physiol., 230, 132-137.

SIEGMAN M. J., 1975. The contractile process in smooth muscle, 1-16. In FRIEDMAN M. H. F., Functions of the stomach and intestine. Univ. Park Press, London.

STEMPER T. J., COOKE A. R., 1975. Gastric emptying and its relationship to antral contractile activity. Gastroenterology, 69, 649-653.

SUMMERS R. W., HELM J., CHRISTENSEN J., 1976. Intestinal propulsion in the dog. Its relations to food intake and the migratory myoelectric complex. Gastroenterology, 70, 753-758.

SZURSZEWSKI J. H., 1969. A migrating electric complex of the canine small intestine. Am. J. Physiol., 217, 1757-1763.

SZURSZEWSKI J. H., 1973. Recording of electrical activity of smooth muscle by means of the sucrose gap 409-425. Proc. 4th int. Symp. Gastrointest. Motility. Mitchell Press, Vancouver (Canada).

SZURSZEWSKI J. H., 1975. Mechanism of action of pentagastrin 'and acetylcholine on the longitudinal muscle of the canine antrum, J. Physiol., Lond., 252, 335-361.

SZURSZEWSKI J. H., 1977. Modulation of smooth muscle by nervous activity : A review and hypothesis. Fed. Proc., 36, 2456-2461.

SZURSZEWSKI J. H., CODE C. F., 1968. Activity fronts of the canine small intestine (abstr.). Gastroenterology, 54, 1304.

TASAKA K., FARRAR J. T., 1976. Intraluminal pressure of the small intestine of the unanesthetized dog. Pflügers Arch., 364, 35-44.

TAYLOR G. S., DANIEL E. E., TOMITA T., 1975. Origin and mechanism of infestinal slow waves, 102-106. In 5th int. Symp. Gostrointest. Motility. VANTRAPPEN G., AGG H. O., Typoff-Press, Herentals (Belg.).

THOMAS J. E., 1955. The gradient theory versus the reflex theory of intestinal peristalsis. Am. J. Gas. troenterol., 23, 13-25.

THOMAS J. E., CRIDER J. O., 1935. Rhythmic changes in duodenal motility associated with gastric peristalsis. Amer. J. Physiol., 111, 124-129.

WEEMS W. A., SEYGAL G. E., 1978. Evaluation of the myogenic ability of cat ileum to do propulsive work on a fluid load (abstr.). Gastroenterology, 74, 1110.

WEISBRODT N. W., 1974. Gastrointestinal motility. MTP Int Rev. Sci., Physiol. Ser. one, 4, $139-181$. 